\title{
The Properties of Planck Galactic Cold Clumps in the L1495 Dark Cloud
}

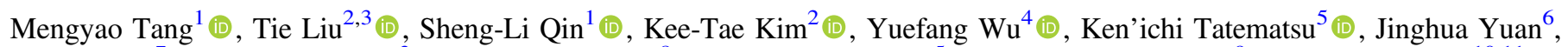 \\ Ke Wang ${ }^{7}$ (1) , Harriet Parsons ${ }^{3}$ (1), Patrick M. Koch ${ }^{8}$ (1), Patricio Sanhueza ${ }^{5}$ (1) D. Ward-Thompson ${ }^{9}$ (10), L. Viktor Tóth ${ }^{10,11}$, \\ Archana Soam ${ }^{2}$, Chang Won Lee ${ }^{2,12}$, David Eden ${ }^{13}$, James Di Francesco ${ }^{14,15}{ }^{(\mathbb{D}}$, Jonathan Rawlings ${ }^{16}$, Mark G. Rawlings ${ }^{3}$, \\ Julien Montillaud $^{17}$, Chuan-Peng Zhang ${ }^{17}$ (D), and M. R. Cunningham ${ }^{18}$ \\ ${ }^{1}$ Department of Astronomy, Yunnan University, and Key Laboratory of Astroparticle Physics of Yunnan Province, Kunming, 650091, People's Republic of China \\ mengyao_tang@yeah.net, slqin@bao.ac.cn \\ ${ }^{2}$ Korea Astronomy and Space Science Institute, 776 Daedeokdaero, Yuseong-gu, Daejeon 34055, Republic of Korea; liu@kasi.re.kr, ktkim@kasi.re.kr \\ ${ }^{3}$ East Asian Observatory, 660 N. A'ohoku Place, Hilo, HI 96720, USA \\ ${ }^{4}$ Department of Astronomy, Peking University, 100871, Beijing, People's Republic of China; ywu@pku.edu.cn \\ ${ }^{5}$ National Astronomical Observatory of Japan, National Institutes of Natural Sciences, 2-21-1 Osawa, Mitaka, Tokyo 181-8588, Japan \\ ${ }^{6}$ National Astronomical Observatories, Chinese Academy of Sciences, Beijing, 100101, People's Republic of China \\ ${ }^{7}$ European Southern Observatory, Karl-Schwarzschild-Str. 2, D-85748 Garching bei München, Germany \\ ${ }^{8}$ Institute of Astronomy and Astrophysics, Academia Sinica, Taipei, Taiwan \\ 9 Jeremiah Horrocks Institute for Mathematics, Physics \& Astronomy, University of Central Lancashire, Preston PR1 2HE, UK \\ ${ }^{10}$ Eötvös Loránd University, Department of Astronomy, Pázmány Péter sétány 1/A, H-1117, Budapest, Hungary \\ ${ }^{11}$ Konkoly Observatory of the Hungarian Academy of Sciences, H-1121 Budapest, Konkoly Thege Miklós út 15-17, Hungary \\ ${ }^{12}$ University of Science \& Technology, 176 Gajeong-dong, Yuseong-gu, Daejeon, Republic of Korea \\ ${ }^{13}$ Astrophysics Research Institute, Liverpool John Moores University, IC2, Liverpool Science Park, 146 Brownlow Hill, Liverpool L3 5RF, UK \\ ${ }^{14}$ NRC Herzberg Astronomy and Astrophysics, 5071 West Saanich Road, Victoria, BC V9E 2E7, Canada \\ ${ }^{15}$ Department of Physics and Astronomy, University of Victoria, Victoria, BC V8P 5C2, Canada \\ ${ }^{16}$ Department of Physics and Astronomy, University College London, Gower Street, London, WC1E 6BT, UK \\ ${ }^{17}$ Institut UTINAM-UMR 6213 - CNRS-Univ Bourgogne Franche Comte, France \\ ${ }^{18}$ School of Physics, University of New South Wales, Sydney, NSW 2052, Australia \\ Received 2017 September 5; revised 2018 January 26; accepted 2018 February 5; published 2018 April 2
}

\begin{abstract}
Planck Galactic Cold Clumps (PGCCs) possibly represent the early stages of star formation. To understand better the properties of PGCCs, we studied 16 PGCCs in the L1495 cloud with molecular lines and continuum data from Herschel, JCMT/SCUBA-2, and the PMO $13.7 \mathrm{~m}$ telescope. Thirty dense cores were identified in 16 PGCCs from 2D Gaussian fitting. The dense cores have dust temperatures of $T_{\mathrm{d}}=11-14 \mathrm{~K}$, and $\mathrm{H}_{2}$ column densities of $N_{\mathrm{H}_{2}}$ $=(0.36-2.5) \times 10^{22} \mathrm{~cm}^{-2}$. We found that not all PGCCs contain prestellar objects. In general, the dense cores in PGCCs are usually at their earliest evolutionary stages. All the dense cores have non-thermal velocity dispersions larger than the thermal velocity dispersions from molecular line data, suggesting that the dense cores may be turbulence-dominated. We have calculated the virial parameter $\alpha$ and found that 14 of the dense cores have $\alpha<2$, while 16 of the dense cores have $\alpha>2$. This suggests that some of the dense cores are not bound in the absence of external pressure and magnetic fields. The column density profiles of dense cores were fitted. The sizes of the flat regions and core radii decrease with the evolution of dense cores. $\mathrm{CO}$ depletion was found to occur in all the dense cores, but is more significant in prestellar core candidates than in protostellar or starless cores. The protostellar cores inside the PGCCs are still at a very early evolutionary stage, sharing similar physical and chemical properties with the prestellar core candidates.
\end{abstract}

Key words: ISM: clouds - ISM: individual objects (L1495) - ISM: molecules - stars: formation

Supporting material: figure sets

\section{Introduction}

Stars form in dense cores within clumpy and filamentary molecular clouds (André et al. 2010). The dense cores that have no protostars are known as starless cores. When starless cores become dense enough to be gravitationally bound, they are known as prestellar cores (Ward-Thompson et al. 1994). Then, the prestellar cores collapse to form Class 0 and then Class I protostars. The prestellar cores are compact (with sizes of $0.1 \mathrm{pc}$ or less), cold $\left(T_{\mathrm{k}} \leqslant 15 \mathrm{~K}\right)$, and dense $\left(n_{\mathrm{H}_{2}}>5 \times 10^{4}\right.$ $\mathrm{cm}^{-3}$ ) starless condensations (Caselli 2011). Herschel observations have revealed that more than $70 \%$ of the prestellar cores (and protostars) are embedded in larger, parsec-scale filamentary structures within molecular clouds, which have column densities exceeding a minimum density threshold $\left(\sim 7 \times 10^{21} \mathrm{~cm}^{-2}\right)$ for core formation (André et al. 2014). The properties of prestellar cores, however, are still not well known due to the lack of a large sample from observations at high spatial resolution in continuum and molecular lines. The highfrequency channels of Planck cover the peak thermal emission frequencies of dust colder than $14 \mathrm{~K}$. A total of 13,188 Planck Galactic Cold Clumps (PGCCs) were identified (Planck Collaboration et al. 2016). PGCCs have low dust temperatures of $6-20 \mathrm{~K}$, and smaller line widths, but modest column densities when compared to other kinds of star-forming clouds (Planck Collaboration et al. 2011, 2016; Wu et al. 2012; Liu et al. 2013, 2014). A large fraction of PGCCs seem to be quiescent and unaffected by ongoing star formation activity (Wu et al. 2012; Yuan et al. 2016). Those sources are the prime candidates for probing how prestellar cores form and evolve, and for studying the very early stages of star formation across a wide variety of galactic environments. A large fraction of PGCCs contain YSOs and are forming new stars. As Zahorecz 
et al. (2016) pointed out based on Herschel data, about $25 \%$ of PGCC clumps near the Galactic mid-plane may be massive enough to form high-mass stars and star clusters. About $30 \%$ of the Taurus, Auriga, Perseus, and California PGCC clumps have associated YSOs (Tóth et al. 2016). Studying the correlation between the Planck ECC clumps (Planck Collaboration et al. 2011) and the AKARI YSOs (Tóth et al. 2014) revealed that 163 of the 915 clumps $(17.8 \%)$ have at least one associated YSO within a radius equal to the semimajor axis of the clump.

The properties of the PGCCs are still not well understood due to the relatively poor resolution of the Planck telescope. To understand better the properties of PGCCs, we have been conducting a series of follow-up surveys toward the PGCCs with several ground-based telescopes such as the PMO (Purple Mountain Observatory) $13.7 \mathrm{~m}$, the TRAO (Taeduk Radio Astronomy Observatory) $14 \mathrm{~m}$, the JCMT (James Clerk Maxwell Telescope) $15 \mathrm{~m}$, and the NRO (Nobeyama Radio Observatory) $45 \mathrm{~m}$ telescopes (Liu et al. 2012, 2013, 2014, 2016; Wu et al. 2012; Meng et al. 2013; Yuan et al. 2016; Zhang et al. 2016; Kim et al. 2017; Juvela et al. 2017; Tatematsu et al. 2017). Using the SCUBA-2 submillimeter camera on the JCMT $15 \mathrm{~m}$ telescope, we have been carrying out a legacy survey toward 1000 PGCCs in the $850 \mu \mathrm{m}$ continuum, namely "SCOPE: SCUBA-2 Continuum Observations of Pre-protostellar Evolution" (Liu et al. 2018). Thousands of dense cores have been identified by the "SCOPE" survey, and most of them are either starless cores or protostellar cores with very young (Class 0/I) objects.

The L1495 cloud is located at $\sim 140$ pc (Straizys \& Meistas 1980; Elias 1987; Kenyon et al. 1994; Loinard et al. 2008), and is representative of a predominantly non-clustered low-mass star formation region. Torres et al. (2012) argued that L1495 is located at a nearer distance of $131.4 \mathrm{pc}$, but here we retain a distance of $140 \mathrm{pc}$ to be consistent with other studies toward L1495. The Taurus molecular cloud complex consists of clusters of cold clouds (Tóth et al. 2017), and is dominated by two roughly parallel filamentary structures, as seen, for example, in the Herschel Gould Belt Survey (André et al. 2010), L1495 being the most prominent filament of them. A large number of cold dense cores exist in this region (Schmalzl et al. 2010; Hacar et al. 2013; Marsh et al. 2016; Ward-Thompson et al. 2016). $\mathrm{N}_{2} \mathrm{H}^{+}$ observations toward L1495 performed by Hacar et al. (2013) suggest that at least 19 dense cores are embedded in the filamentary cloud. Other studies such as $\mathrm{H}^{13} \mathrm{CO}^{+}$performed by Onishi et al. (2002), $\mathrm{NH}_{3}$ by Seo et al. (2015), and continuum studies (Kirk et al. 2013; Marsh et al. 2014, 2016; WardThompson et al. 2016), have suggested that L1495 cloud is composed of active low-mass star-forming regions. The properties of those dense cores in L1495, however, have not yet been fully investigated. In this paper, we aim to characterize the properties of a small sample of 16 PGCCs in the L1495 cloud, with data from Herschel, PMO, and SCUBA-2. The evolutionary stages, masses, density structures, and $\mathrm{CO}$ gas depletion of the dense cores inside the PGCCs will be investigated in detail. These studies will deepen our understandings of the physical and chemical properties of those dense cores in L1495, as well as PGCCs in general.

The paper is organized as follows. In Section 2, we present the observed data. In Section 3, we describe the observational results. In Section 4, we discuss the properties of dense cores. We summarize our findings and provide a general conclusion in Section 5.

\section{Observations and Data}

\subsection{Herschel Data}

The Herschel Space Observatory is a $3.5 \mathrm{~m}$ diameter telescope that operated in the far-infrared and submillimeter regimes (Pilbratt et al. 2010). The Herschel data of L1495 used in this paper are part of the Herschel Gould Belt Survey (André et al. 2010) and were presented by Marsh et al. (2016). The details of the observations and data reduction can be seen in André et al. (2010) and Marsh et al. (2016). In this paper, we directly used the column density and dust temperature maps of L1495 from Marsh et al. (2016), which have a 18" angular resolution. The column density map and dust temperature map were derived from SPIRE continuum data, by fitting pixel-bypixel SEDs using (Hildebrand 1983)

$$
F_{\nu}=\frac{N_{\mathrm{H}_{2}} m_{\mathrm{H}} \mu A B_{\nu}(T) \kappa_{\nu}}{D^{2}},
$$

where $F_{\nu}$ is the flux density at frequency $\nu$ and $B_{\nu}$ is the Planck Function. $m_{\mathrm{H}}$ is the atomic hydrogen mass and $\mu$ is the mean weight of molecules taken as 2.8 (Kauffmann et al. 2008), and $A$ is the area of each pixel. A dust mass opacity $0.144 \mathrm{~cm}^{2} \mathrm{~g}^{-1}$ has been derived for $\kappa_{\nu}=0.1 \times\left(300 / \lambda_{[\mu \mathrm{m}]}\right)^{2}$ at $250 \mu \mathrm{m}$ and a gas-to-dust mass ratio of 100 is adopted (Hildebrand 1983; Marsh et al. 2016). The pixel size is $6^{\prime \prime}$. The distance $D$ is 140 pc (Straizys \& Meistas 1980; Elias 1987; Kenyon et al. 1994; Loinard et al. 2008). Therefore, only $T$ and $N_{\mathrm{H}_{2}}$ are free parameters to fit. The SED fitting and maps were made by Marsh et al. (2016).

\subsection{SCUBA-2 Data}

The Submillimetre Common User Bolometer Array 2 (SCUBA-2) is a bolometer detector operating on the JCMT $15 \mathrm{~m}$ telescope with 5120 bolometers in each of two simultaneous imaging bands centred at $450 \mu \mathrm{m}$ and $850 \mu \mathrm{m}$ (Holland et al. 2013). In this paper we use SCUBA-2 data toward eight of the PGCCs from both the SCOPE survey (M16AL003 and M15BI061; PI: Tie Liu) and $\mathrm{CADC}^{19}$ archival data. The archival data for G170.26-16.02 (MJLSG37) were actually taken by the SCUBA-2 Gould Belt Legacy Survey (Ward-Thompson et al. 2007; Buckle et al. 2015). The observations and programs associated with these eight PGCCs are provided in Table 1. The data were observed primarily using the CV Daisy mode. The CV Daisy is designed for small compact sources, providing a deep $3^{\prime}$ region in the center of the map but coverage out to beyond $12^{\prime}$ (Bintley et al. 2014). We used CV Daisy mode in the SCOPE survey because this mode is more efficient to quickly survey a large sample. The aim of the SCOPE survey is to detect dense condensations inside PGCCs. All the SCUBA-2 $850 \mu \mathrm{m}$ continuum data were reduced using an iterative mapmaking technique (Chapin et al. 2013; Currie et al. 2014; Mairs et al. 2015). Specifically, the data were all run with the same reduction tailored for compact sources, filtering out scales larger than $200^{\prime \prime}$ on a $4^{\prime \prime}$ pixel scale. A Flux Conversion Factor (FCF) of $554 \mathrm{Jy} / \mathrm{pW} /$ beam was used to convert data from $\mathrm{pW}$ to $\mathrm{Jy} /$ beam (Liu et al. 2018). The FCF in this paper is higher than the canonical value derived by Dempsey et al. (2013). This higher value reflects the impact of the data reduction technique and pixel size used by the authors. The pixel size used in the reduction of a

\footnotetext{
19 http://www.cadc-ccda.hia-iha.nrc-cnrc.gc.ca/
} 
Table 1

The SCUBA-2 Observations

\begin{tabular}{|c|c|c|c|c|}
\hline Name & Project ID & UT & obs Number(s) & $\mathrm{rms}^{\mathrm{a}}(\mathrm{mJy} /$ beam $)$ \\
\hline G168.13-16.39 & M16AL003 & 2015 Dec 17 & 33 & 11.93 \\
\hline G168.72-15.48 & M16AL003 & 2016 Jan 13 & 13 & 7.18 \\
\hline G169.76-16.15 & M16AL003 & 2015 Dec 27 & 36 & 12.37 \\
\hline G170.26-16.02 & MJLSG37 ${ }^{\circ}$ & 2014 Nov 16 & 21,25 and 24 & 12.50 \\
\hline G171.49-14.90 & M16AL003 & 2016 Jan 17 & 20 & 9.24 \\
\hline G171.80-15.32 & M15BI061 ${ }^{\mathrm{d}}$ & 2015 Sep 28 & 15 & 9.22 \\
\hline
\end{tabular}

Notes.

The rms in the final reduced map.

b PGCCs G169.76-16.15 and G170.00-16.14 are covered within single observations.

c These observations were PONG 1800 observations observed as part of the JCMT Gould Belt Survey (Ward-Thompson et al. 2007; Buckle et al. 2015).

d M15BI061 is a pilot study of the SCOPE survey.

e JCMT calibration observation of object DGTau.

calibrator can have a significant effect on the FCF derived. The effect is different for both the beam and aperture FCFs, and also for different calibrators (Dempsey et al. 2013). Therefore, we derived a new FCF for the SCOPE survey for a default pixel size of $4^{\prime \prime}$. We note that the flux calibration uncertainty in SCOPE survey is less than $10 \%$ at the $850 \mu \mathrm{m}$ band. The archival data for G170.26-16.02 and G171.91-15.65 were calibrated with a FCF of $537 \mathrm{Jy} / \mathrm{pW} /$ beam. The FCF used for those archival data are consistent with the values $(528 \mathrm{Jy} / \mathrm{pW} /$ beam for G170.26-16.02 and $526 \mathrm{Jy} / \mathrm{pW} /$ beam for G171.91-15.65) derived from the calibrators observed at the same time. We note that G170.2616.02 was observed with Pong 1800 mode. The filtering out scale for G170.26-16.02 data reduction is 600". However, a different filtering out scale would not affect the core properties because the core sizes in G170.26-16.02 are much smaller than 100".

\subsection{PMO 13.7 m Telescope Data}

Observations of the 16 PGCCs in L1495 in the ${ }^{12} \mathrm{CO}(1-0),{ }^{13} \mathrm{CO}(1-0)$, and $\mathrm{C}^{18} \mathrm{O}(1-0)$ lines were performed with the PMO $13.7 \mathrm{~m}$ telescope between 2011 January and May. The 9-beam array receiver system in double-sideband (DSB) mode was used as the front end (Shan et al. 2012). The ${ }^{12} \mathrm{CO}(1-0)$ line was observed in the upper sideband and both ${ }^{13} \mathrm{CO}(1-0)$ and $\mathrm{C}^{18} \mathrm{O}(1-0)$ were observed simultaneously in the lower sideband. The half-power beam width was $56^{\prime \prime}$ with a main beam efficiency of $50 \%$. The pointing and tracking accuracies were both better than $5^{\prime \prime}$. The spectral resolution was $\sim 61 \mathrm{KHz}$, corresponding to a velocity resolution of $0.16 \mathrm{~km} \mathrm{~s}^{-1}$.

The on-the-fly (OTF) observing mode was used. OTF data were converted to three-dimensional datacubes with a grid spacing of 30". Then, we used MIRIAD (Sault et al. 1995) for further analyses. A description of the analyses of the OTF data can be found in Liu et al. (2012) and Meng et al. (2013).

\section{Results}

The distributions of the 16 PGCCs are shown in Figure 1, which clearly shows the filamentary structure in L1495. The names and coordinates of the 16 PGCCs are listed in Table 2.

\subsection{Identification and Classification of Dense Cores}

We identified dense cores within the observed PGCCs based on the column density $\left(N_{\mathrm{H}_{2}}\right)$ maps derived from Herschel data by eye. We did not apply any core finder algorithm because the emission peaks in the Taurus PGCCs can be very easily identified by eye. Then, the identified dense cores were fitted with a 2D Gaussian to get the physical parameters (e.g., size, temperature, density). We mainly focus on the dense cores with core-averaged column densities larger than $3 \times 10^{21} \mathrm{~cm}^{-2}$. The density threshold we used is about half of the minimum density threshold $\left(\sim 7 \times 10^{21} \mathrm{~cm}^{-2}\right)$ for core formation discovered in Herschel observations (André et al. 2014). Their peak column densities are also larger than $\sim 7 \times 10^{21} \mathrm{~cm}^{-2}$. In total, the 30 most reliable dense cores with mean column densities larger than $3 \times 10^{21} \mathrm{~cm}^{-2}$ are identified in 16 clumps from Herschel data.

As mentioned in Section 1, dense cores that have no protostars are classified as starless cores. When starless cores become dense enough to be gravitationally bound, they become prestellar cores (Ward-Thompson et al. 1994). Then, the dense cores will collapse to form Class 0 and then Class I protostars.

To investigate how core properties change with evolution, we classify the dense cores into starless, prestellar, and protostellar categories. The presence of a Herschel $70 \mu \mathrm{m}$ source is a signpost of ongoing star formation (Könyves et al. 2015). Therefore, those dense cores with $70 \mu \mathrm{m}$ emission are protostellar. In contrast, those with no $70 \mu \mathrm{m}$ emission are starless. As an example, Figure 2 presents the Herschel column density map (in contours) overlaid on Herschel $70 \mu \mathrm{m}$ emission map for a representative source PGCC G171.91-15.65. The cores "H1" and "H2" in G171.91-15.65 are associated with protostars, while "H3" is starless. In this paper, we only show images for G171.9115.65. the images for other pgccs are shown in the figure set.

Prestellar cores are gravitationally bound starless cores and have higher densities than unbound starless cores. Many factors, like gravity, turbulence, magnetic field, external pressure, and even bulk motions, can affect the stability of dense cores. Therefore, it is hard to tell whether or not a starless core is gravitationally bound based on present line data. The dense starless cores detected by SCUBA-2, however, have higher densities than other starless cores (e.g., those without SCUBA-2 detection) and should be good candidates for prestellar cores (Ward-Thompson et al. 2016). Therefore, in this paper, we classify the starless cores with SCUBA-2 detection as prestellar core candidates. Hereafter, "starless cores" stand for those starless cores without SCUBA-2 detection. In total, we identify 9 protostellar cores, 6 prestellar core candidates, and 15 starless cores. The starless cores, prestellar core 
Table 2

The Coordinates of Observed Planck Cold Clumps

\begin{tabular}{|c|c|c|c|c|c|c|c|}
\hline Name & $\begin{array}{c}\text { Glon } \\
\left({ }^{\circ}\right)\end{array}$ & $\begin{array}{c}\text { Glat } \\
\left({ }^{\circ}\right)\end{array}$ & $\begin{array}{l}\text { R.A.(J2000) } \\
\quad(\text { h m s })\end{array}$ & $\begin{array}{l}\text { Decl.(J2000) } \\
\quad(\mathrm{d} \mathrm{m} \mathrm{s})\end{array}$ & $N_{\mathrm{VC}}{ }^{\mathrm{a}}$ & $N_{\text {core }}{ }^{\mathrm{b}}$ & $\overline{N_{\text {condensation }}{ }^{\mathrm{c}}}$ \\
\hline G166.99-15.34 & 166.99217 & -15.346058 & 041342.01 & +294426.31 & 2 & 1 & 0 \\
\hline G167.23-15.32 & 167.23387 & -15.326718 & 041430.92 & +293516.09 & 1 & 1 & 0 \\
\hline G168.00-15.69 & 168.00291 & -15.694481 & 041540.46 & +284800.55 & 1 & 1 & 0 \\
\hline G168.13-16.39 & 168.13475 & -16.393131 & 041347.56 & +281322.16 & 2 & 2 & 3 \\
\hline G168.72-15.48 & 168.72801 & -15.481486 & 041834.58 & +282634.64 & 1 & 2 & 3 \\
\hline G169.43-16.17 & 169.43114 & -16.179394 & 041823.08 & +272818.97 & 2 & 2 & 0 \\
\hline G169.76-16.15 & 169.76073 & -16.159975 & 041925.47 & +271516.21 & 2 & 5 & 3 \\
\hline G170.00-16.14 & 170.00243 & -16.140558 & 042012.04 & +270552.89 & 2 & 2 & 2 \\
\hline G170.13-16.06 & 170.13426 & -16.062908 & 042050.61 & +270329.08 & 2 & 1 & 0 \\
\hline G170.26-16.02 & 170.2661 & -16.024094 & 042121.47 & +265929.26 & 1 & 2 & 3 \\
\hline G170.83-15.90 & 170.83739 & -15.907699 & 042324.42 & +263955.87 & 2 & 2 & 0 \\
\hline G170.99-15.81 & 170.9912 & -15.810754 & 042410.41 & +263716.68 & 1 & 1 & 0 \\
\hline G171.49-14.90 & 171.49657 & -14.901693 & 042839.71 & +265156.81 & 2 & 1 & 2 \\
\hline G171.80-15.32 & 171.80418 & -15.326718 & 042807.26 & +262141.65 & 1 & 3 & 3 \\
\hline G171.91-15.65 & 171.91405 & -15.655738 & 042720.24 & +260350.27 & 1 & 3 & 2 \\
\hline G172.06-15.21 & 172.06786 & -15.210718 & 042915.63 & +261452.01 & 1 & 1 & 0 \\
\hline
\end{tabular}

Notes.

${ }^{\text {a }} N_{\mathrm{VC}}$ represents the number of velocity components.

${ }^{\mathrm{b}} N_{\text {core }}$ represents the number of dense cores detected by Herschel.

${ }^{\mathrm{c}} N_{\text {condensation }}$ represents the number of dense condensations detected by SCUBA-2.

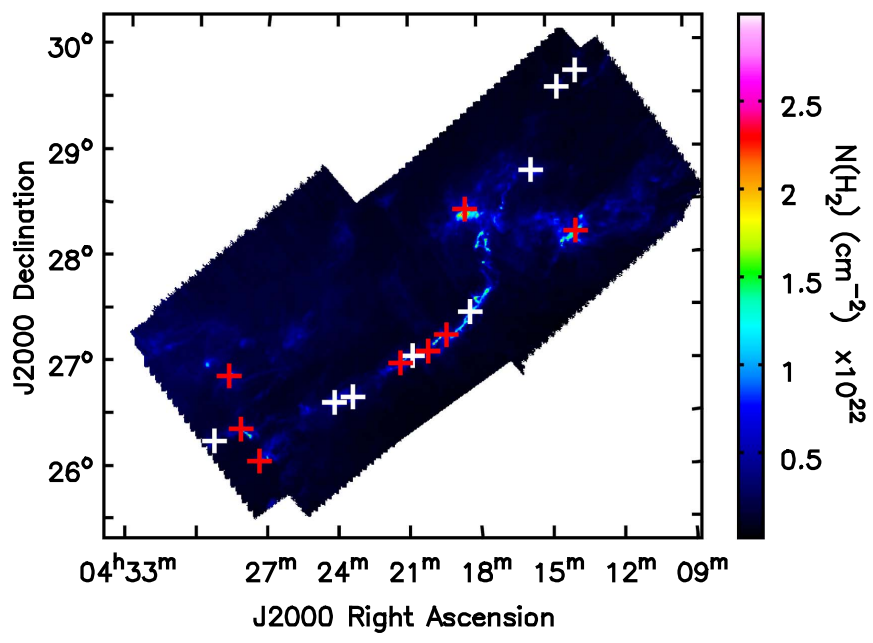

Figure 1. Spatial distribution of the observed 16 PGCCs, which are marked with crosses. The red cross represents the PGCC with SCUBA-2 detection, while the white cross represents the PGCC without SCUBA-2 detection. The color image is the Herschel $\mathrm{H}_{2}$ column density map, which was derived from Herschel continuum data by fitting SEDs pixel-by-pixel (Marsh et al. 2016). An obvious filamentary structure in L1495 is visible.

candidates, and protostellar cores are marked with 1,2 , or 3 in column 10 of Table 3 , respectively.

\subsection{Herschel Images}

The Herschel column density distributions and dust temperature map are shown in contours and colors in panel (a) of Figure 3, respectively. The coordinates and FWHM deconvolved major and minor axes of the dense cores ( $a$ and $b$ ) were obtained by $2 \mathrm{D}$ Gaussian fits. The effective radius is $R=\sqrt{a b} D$, where $D$ is the distance. The core-averaged $N_{\mathrm{H}_{2}}$ (Herschel) and $T_{\mathrm{d}}$ of dense cores are derived within a 2 FWHM (or $2 R$ ) area, and presented in Table 3. The core-averaged $N_{\mathrm{H}_{2}}$ (Herschel) of the dense cores

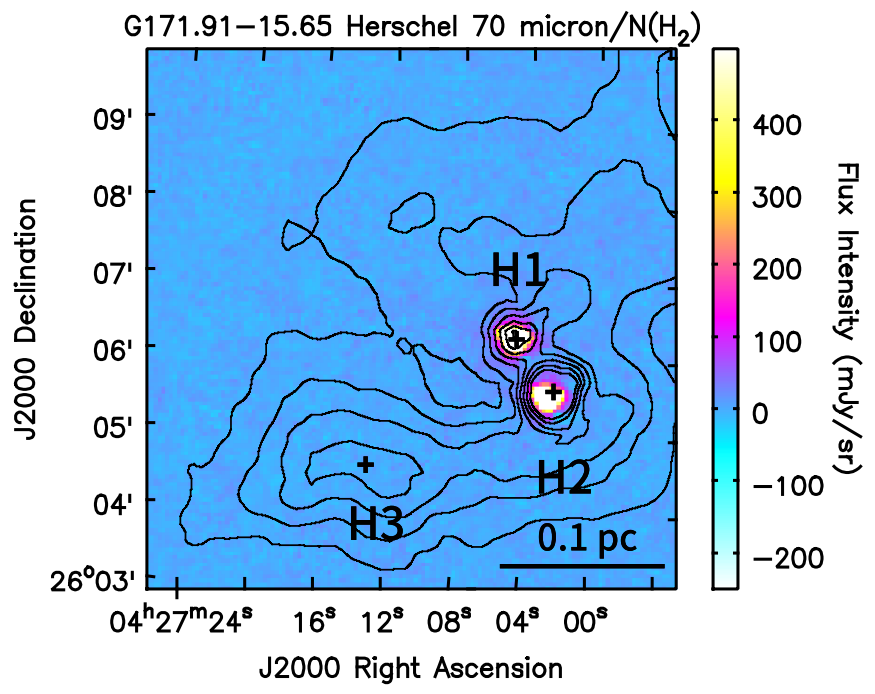

Figure 2. The Herschel column density map has been overlaid on a Herschel $70 \mu \mathrm{m}$ continuum emission map for the representative source PGCC G171.91-15.65. The images for other sources are shown in the figure set. The contours represent the column density distribution from Herschel. The contour levels are from $30 \%$ to $90 \%$ of the peak value, in steps of $10 \%$. The Herschel $70 \mu \mathrm{m}$ continua are shown in color. The protostellar cores " $\mathrm{H} 1$ " and "H2" are associated with $70 \mu \mathrm{m}$ sources, while the starless core "H3" is not associated with any $70 \mu \mathrm{m}$ source. The images for other sources are shown in the figure set.

(The complete figure set ( 8 images) is available.)

range from $3.6( \pm 1.0) \times 10^{21}$ to $2.5( \pm 0.6) \times 10^{22} \mathrm{~cm}^{-2}$. The core-averaged dust temperatures of the dense cores range from $11.1( \pm 0.4)$ to $13.8( \pm 0.4) \mathrm{K}$. If we assume that the dense core is a sphere with a radius of $R$, the volume density of the core is roughly $n_{\mathrm{H}_{2}}=N_{\mathrm{H}_{2}}^{\text {peak }} / 2 R$, where $N_{\mathrm{H}_{2}}^{\text {peak }}$ is the peak column density of a dense core. The volume densities $\left(n_{\mathrm{H}_{2}}\right)$ of the dense cores range from $5.7( \pm 0.9) \times 10^{3} \mathrm{~cm}^{-3}$ to $7.9( \pm 1.4) \times 10^{4} \mathrm{~cm}^{-3}$. The 
Table 3

The Parameters Derived from Herschel Data

\begin{tabular}{|c|c|c|c|c|c|c|c|c|c|c|c|}
\hline Name & $\begin{array}{l}\text { Coordinate(J2000) } \\
\text { (R.A., Decl.) }\end{array}$ & $\begin{array}{l}\text { Deconvolved Size } \\
\text { (“×”) }\end{array}$ & $\begin{array}{l}\text { P.A }{ }^{a} \\
\left({ }^{\circ}\right)\end{array}$ & $\begin{array}{c}N_{\mathrm{H}_{2}}(\text { Herschel }) \\
\left(10^{22} \mathrm{~cm}^{-2}\right)\end{array}$ & $\begin{array}{l}N_{\mathrm{H}_{2}}^{\text {peak }}(\text { Herschel }) \\
\quad\left(10^{22} \mathrm{~cm}^{-2}\right)\end{array}$ & $\begin{array}{c}T_{d} \\
(\mathrm{~K})\end{array}$ & $\begin{array}{c}R^{\mathrm{b}} \\
(\mathrm{pc})\end{array}$ & $\begin{array}{c}n_{\mathrm{H}_{2}} \\
\left(10^{4} \mathrm{~cm}^{-3}\right)\end{array}$ & $\begin{array}{l}M_{\text {core }} \\
\left(M_{\odot}\right)\end{array}$ & Classification $^{\mathrm{c}}$ & Remark $^{\mathrm{d}}$ \\
\hline G166.99-15.34-H1 & $(4: 13: 41.951,+29: 43: 43.811)$ & $214 \times 176$ & -65 & $0.36( \pm 0.10)$ & 0.62 & $13.1( \pm 0.4)$ & 0.11 & $0.90( \pm 0.14)$ & $3.6( \pm 0.6)$ & 1 & $\cdots$ \\
\hline G167.23-15.32-H1 & $(4: 14: 29.120,+29: 35: 14.313)$ & $233 \times 112$ & 78 & $0.75( \pm 0.24)$ & 1.19 & $12.5( \pm 0.4)$ & 0.11 & $1.75( \pm 0.35)$ & $6.7( \pm 1.3)$ & 1 & $\cdots$ \\
\hline G168.00-15.69-H1 & $(4: 15: 35.466,+28: 47: 23.381)$ & $480 \times 307$ & -86 & $0.59( \pm 0.14)$ & 0.92 & $12.7( \pm 0.3)$ & 0.26 & $0.57( \pm 0.09)$ & $29.2( \pm 4.4)$ & 1 & $\cdots$ \\
\hline G168.13-16.39-H1 & $(4: 13: 49.122,+28: 12: 31.360)$ & $382 \times 164$ & -40 & $1.53( \pm 0.39)$ & 2.54 & $11.9( \pm 0.3)$ & 0.17 & $2.42( \pm 0.37)$ & $34.6( \pm 5.3)$ & 3 & S1 \\
\hline G168.13-16.39-H2 & $(4: 14: 08.510,+28: 09: 24.391)$ & $627 \times 164$ & -40 & $1.33( \pm 0.14)$ & 1.64 & $11.6( \pm 0.2)$ & 0.22 & $1.22( \pm 0.11)$ & $36.4( \pm 3.1)$ & 3 & . \\
\hline G168.72-15.48-H1 & $(4: 18: 33.568,+28: 26: 56.805)$ & $394 \times 158$ & -6 & $1.40( \pm 0.22)$ & 1.97 & $11.9( \pm 0.3)$ & 0.17 & $1.89( \pm 0.21)$ & $26.5( \pm 3.0)$ & 1 & \\
\hline G168.72-15.48-H2 & $(4: 18: 38.298,+28: 23: 22.959)$ & $251 \times 169$ & -85 & $2.46( \pm 0.60)$ & 4.13 & $13.2( \pm 1.0)$ & 0.14 & $4.78( \pm 0.70)$ & $37.8( \pm 5.5)$ & 2 & S1 \\
\hline G169.43-16.17-H1 & $(4: 18: 20.859,+27: 28: 27.755)$ & $616 \times 152$ & -43 & $1.05( \pm 0.23)$ & 1.49 & $11.9( \pm 0.3)$ & 0.21 & $1.16( \pm 0.18)$ & $30.3( \pm 4.7)$ & 1 & $\ldots$ \\
\hline G169.43-16.17-H2 & $(4: 18: 36.843,+27: 22: 20.914)$ & $309 \times 137$ & -8 & $0.99( \pm 0.18)$ & 1.35 & $11.8( \pm 0.3)$ & 0.14 & $1.57( \pm 0.21)$ & $12.3( \pm 1.7)$ & 1 & $\cdots$ \\
\hline G169.76-16.15-H1 & $(4: 19: 01.227,+27: 16: 50.760)$ & $215 \times 119$ & -27 & $0.97( \pm 0.12)$ & 1.17 & $11.9( \pm 0.2)$ & 0.11 & $1.74( \pm 0.18)$ & $6.5( \pm 0.7)$ & 1 & $\ldots$ \\
\hline G169.76-16.15-H2 & $(4: 19: 12.104,+27: 13: 53.438)$ & $232 \times 129$ & -37 & $0.95( \pm 0.09)$ & 1.09 & $12.2( \pm 0.1)$ & 0.12 & $1.51( \pm 0.12)$ & $7.1( \pm 0.6)$ & 1 & $\cdots$ \\
\hline G169.76-16.15-H3 & $(4: 19: 24.655,+27: 15: 05.860)$ & $206 \times 124$ & -48 & $1.05( \pm 0.26)$ & 1.70 & $12.1( \pm 0.3)$ & 0.11 & $2.53( \pm 0.39)$ & $9.4( \pm 1.5)$ & 2 & S3 and 21 \\
\hline G169.76-16.15-H4 & $(4: 19: 37.299,+27: 15: 19.802)$ & $170 \times 144$ & -69 & $1.11( \pm 0.24)$ & 1.66 & $11.8( \pm 0.3)$ & 0.11 & $2.53( \pm 0.37)$ & $8.8( \pm 1.3)$ & 1 & 22 \\
\hline G169.76-16.15-H5 & $(4: 19: 42.843,+27: 13: 32.947)$ & $142 \times 78$ & -75 & $1.13( \pm 0.35)$ & 1.84 & $12.0( \pm 0.3)$ & 0.072 & $4.17( \pm 0.79)$ & $4.4( \pm 0.8)$ & 3 & S1 and 23 \\
\hline G170.00-16.14-H1 & $(4: 19: 51.580,+27: 11: 33.739)$ & $108 \times 75$ & -35 & $1.24( \pm 0.30)$ & 1.82 & $11.1( \pm 0.3)$ & 0.062 & $4.82( \pm 0.78)$ & $3.2( \pm 0.5)$ & 2 & S2 and 24 \\
\hline G170.00-16.14-H2 & $(4: 19: 58.119,+27: 10: 16.257)$ & $144 \times 79$ & -44 & $1.25( \pm 0.42)$ & 2.32 & $12.2( \pm 0.8)$ & 0.065 & $5.82( \pm 1.05)$ & $4.6( \pm 0.8)$ & 3 & S1 and 25 \\
\hline G170.13-16.06-H1 & $(4: 20: 54.832,+27: 02: 42.492)$ & $400 \times 188$ & -89 & $1.16( \pm 0.26)$ & 1.64 & $11.5( \pm 0.3)$ & 0.19 & $1.43( \pm 0.22)$ & $26.8( \pm 4.3)$ & 1 & 30,31 and 32 \\
\hline G170.26-16.02-H1 & $(4: 21: 12.207,+27: 01: 17.035)$ & $101 \times 50$ & -51 & $0.99( \pm 0.17)$ & 1.22 & $12.7( \pm 0.4)$ & 0.049 & $4.08( \pm 0.56)$ & $1.3( \pm 0.2)$ & 3 & S2 and 34 \\
\hline G170.26-16.02-H2 & $(4: 21: 21.509,+26: 59: 53.620)$ & $192 \times 85$ & -52 & $1.24( \pm 0.38)$ & 2.19 & $11.1( \pm 0.4)$ & 0.087 & $4.08( \pm 0.71)$ & $7.8( \pm 1.4)$ & 2 & S3 and 33 \\
\hline G170.83-15.90-H1 & $(4: 23: 37.832,+26: 40: 21.545)$ & $204 \times 162$ & 48 & $0.53( \pm 0.13)$ & 0.70 & $12.2( \pm 0.3)$ & 0.12 & $0.93( \pm 0.16)$ & $5.0( \pm 0.9)$ & 1 & $\cdots$ \\
\hline G170.83-15.90-H2 & $(4: 23: 29.382,+26: 38: 35.041)$ & $180 \times 110$ & 50 & $0.54( \pm 0.08)$ & 0.94 & $12.2( \pm 0.3)$ & 0.096 & $1.58( \pm 0.13)$ & $4.0( \pm 0.4)$ & 1 & $\cdots$ \\
\hline G170.99-15.81-H1 & $(4: 24: 17.134,+26: 36: 54.550)$ & $669 \times 308$ & -75 & $0.82( \pm 0.18)$ & 1.35 & $11.8( \pm 0.3)$ & 0.31 & $0.71( \pm 0.10)$ & $59.8( \pm 8.1)$ & 1 & 35 and 36 \\
\hline G171.49-14.90-H1 & $(4: 28: 39.189,+26: 51: 43.701)$ & $135 \times 92$ & -25 & $2.12( \pm 0.67)$ & 3.70 & $11.3( \pm 0.4)$ & 0.076 & $7.87( \pm 1.43)$ & $10.0( \pm 1.8)$ & 3 & S1 \\
\hline G171.80-15.32-H1 & $(4: 28: 09.290,+26: 20: 44.816)$ & $148 \times 97$ & -60 & $1.50( \pm 0.51)$ & 2.52 & $11.1( \pm 0.4)$ & 0.082 & $5.01( \pm 1.01)$ & $7.9( \pm 1.6)$ & 2 & S1 and 39 \\
\hline G171.80-15.32-H2 & $(4: 27: 54.940,+26: 19: 17.994)$ & $97 \times 82$ & 70 & $0.89( \pm 0.15)$ & 1.19 & $12.4( \pm 0.7)$ & 0.061 & $3.17( \pm 0.40)$ & $2.0( \pm 0.3)$ & 3 & S2 and 38 \\
\hline G171.80-15.32-H3 & $(4: 27: 48.456,+26: 18: 07.157)$ & $237 \times 122$ & 59 & $1.34( \pm 0.25)$ & 1.81 & $11.1( \pm 0.2)$ & 0.12 & $2.54( \pm 0.32)$ & $11.3( \pm 1.6)$ & 2 & $\mathrm{~S} 3$ and 37 \\
\hline G171.91-15.65-H1 & $(4: 27: 04.261,+26: 06: 22.493)$ & $86 \times 48$ & 36 & $0.78( \pm 0.26)$ & 1.35 & $13.8( \pm 0.4)$ & 0.044 & $4.99( \pm 0.94)$ & $1.2( \pm 0.2)$ & 3 & S1 \\
\hline G171.91-15.65-H2 & $(4: 27: 02.366,+26: 05: 24.059)$ & $90 \times 61$ & 30 & $0.98( \pm 0.39)$ & 2.07 & $13.3( \pm 0.6)$ & 0.051 & $6.63( \pm 1.26)$ & $2.5( \pm 0.5)$ & 3 & S2 \\
\hline G171.91-15.65-H3 & $(4: 27: 13.117,+26: 04: 30.520)$ & $161 \times 125$ & -72 & $0.82( \pm 0.09)$ & 0.98 & $12.3( \pm 0.1)$ & 0.096 & $1.64( \pm 0.16)$ & $4.2( \pm 0.4)$ & 1 & $\ldots$ \\
\hline G172.06-15.21-H1 & $(4: 29: 15.723,+26: 14: 00.580)$ & $215 \times 113$ & -72 & $0.87( \pm 0.26)$ & 1.47 & $12.1( \pm 0.3)$ & 0.11 & $2.24( \pm 0.40)$ & $7.7( \pm 1.4)$ & 1 & \\
\hline
\end{tabular}

Notes.

${ }^{a}$ Position angle of dense cores detected by Herschel; the convention used for measuring angles is east of north.

${ }^{\mathrm{b}}$ The core radii are derived from elliptic Gaussian fitting to Herschel dense cores.

${ }^{\mathrm{c}}$ Classification of dense cores: 1 represents a starless core, 2 represents a prestellar candidate, and 3 represents a protostellar core.

${ }^{\mathrm{d}} \mathrm{S} 1, \mathrm{~S} 2$ or S3 represent the SCUBA-2 counterparts of dense cores. The numbers are source IDs of $\mathrm{NH}_{3}$ counterparts from Seo et al. (2015). 


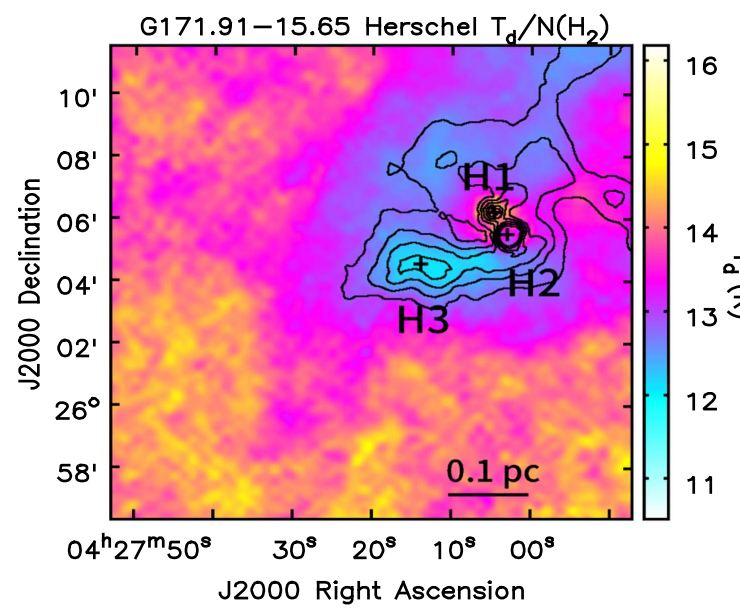

(a)

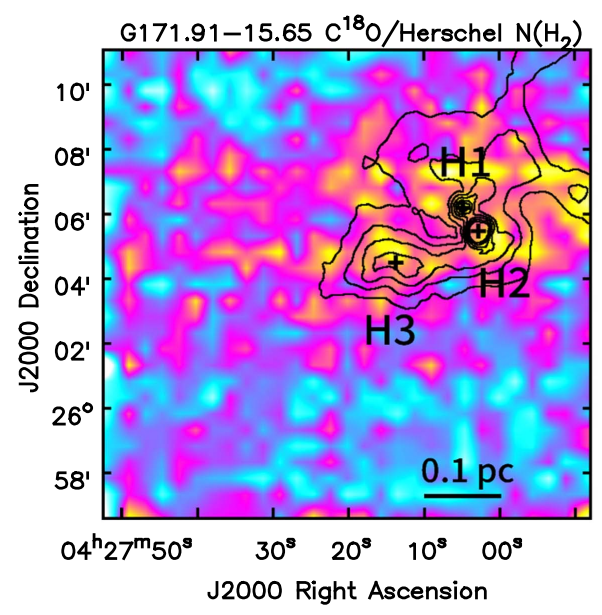

(c)

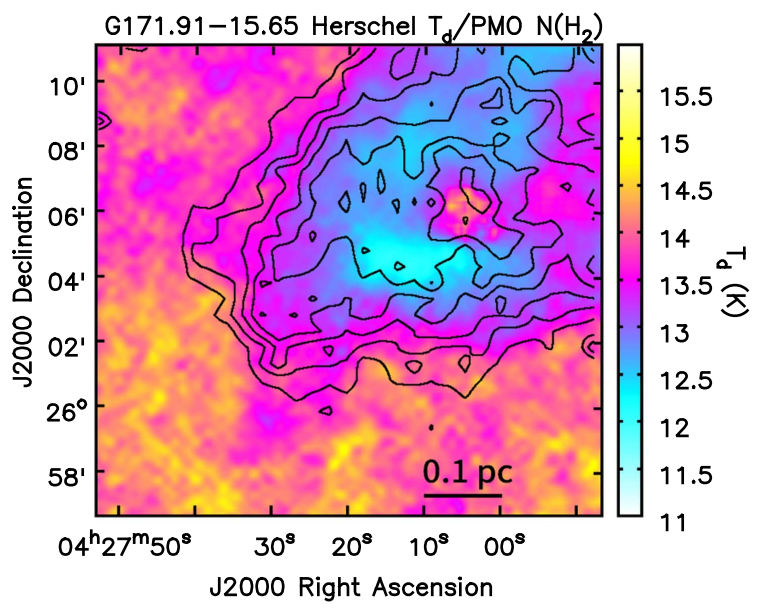

(b)

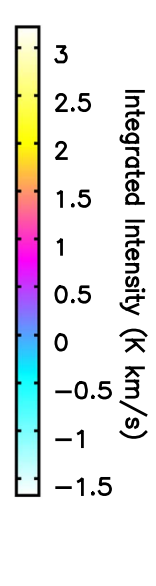

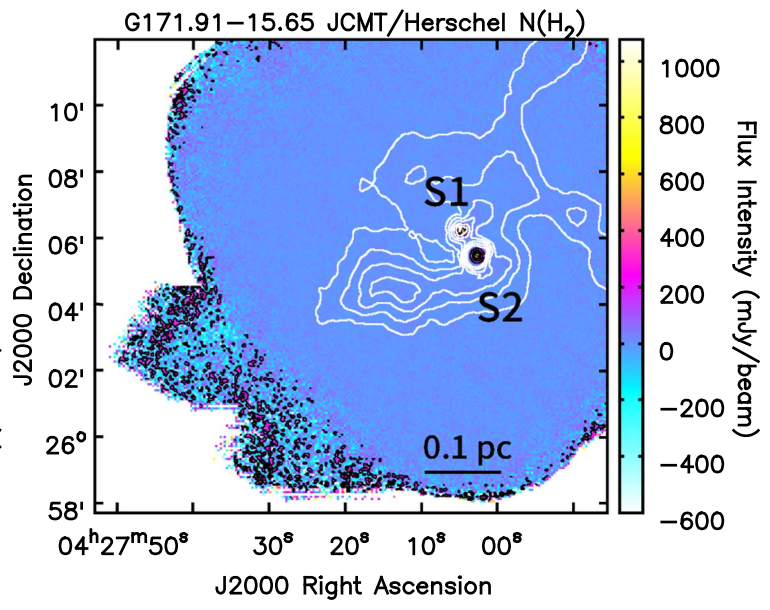

(d)

Figure 3. Panel (a): the Herschel column density map overlaid on the dust temperature map for the representative source PGCC G171.91-15.65. The images for other sources are shown in the figure set. The contours represent the column density distributions from Herschel data. The contour levels are from $30 \%$ to $90 \%$ of the peak value, in steps of $10 \%$. Panel (b): the $\mathrm{PMO}_{2}$ column density map (derived from ${ }^{13} \mathrm{CO}$ data) overlaid onto the dust temperature map for the representative source PGCC G171.91-15.65. The images for other sources are shown in the figure set. The contours represent the column density distribution from PMO data. The contour levels are from $30 \%$ to $90 \%$ of the peak value, in steps of $10 \%$. The dust temperatures are shown in color in K. Panel (c): the Herschel column density map overlaid onto the $\mathrm{C}^{18} \mathrm{O}$ integrated intensity map for the representative source PGCC G171.91-15.65. The images for other sources are shown in the figure set. The contours represent the column density distributions from Herschel data. The contour levels are from $30 \%$ to $90 \%$ of the peak value, in steps of $10 \%$. The integrated intensities of $\mathrm{C}^{18} \mathrm{O}$ data are shown in color in $\mathrm{K} \mathrm{km} \mathrm{s}^{-1}$. Panel (d): the Herschel column density map overlaid on a SCUBA-2 $850 \mu \mathrm{m}$ continuum emission map for the representative source PGCC G171.91-15.65. The images for other sources are shown in the figure set. The white contours represent the Herschel column density distributions. The contour levels are from $30 \%$ to $90 \%$ of the peak value, in steps of $10 \%$. The SCUBA-2 $850 \mu$ m continuum intensities are shown in color and with black contours. The contour levels are from $10 \%$ to $90 \%$ of the peak value, in steps of $20 \%$. The condensations detected by SCUBA-2 are marked with -S1, -S2 or -S3. Only two condensations are detected by SCUBA-2 in PGCC G171.91-15.65 and their sizes are smaller than the dense cores detected by Herschel. The images for other sources are shown in the figure set.

(The complete figure set (16 images) is available.)

masses of the dense cores are estimated as

$$
M_{\text {core }}=\frac{4}{3} \pi R^{3} \cdot n_{\mathrm{H}_{2}} \cdot m_{\mathrm{H}} \cdot \mu,
$$

where $\mu=2.8$ is the mean molecular weight (Kauffmann et al. 2008). $m_{\mathrm{H}}$ is the mass of a $\mathrm{H}$ atom. The core masses $M_{\text {core }}$ are presented in Table 3.

\subsection{SCUBA-2 Continuum Images}

Panel (d) of Figure 3 presents the $850 \mu \mathrm{m}$ map from SCUBA-2 overlaid on a Herschel column density map. The
SCUBA-2 detected condensations are marked with -S1, $-\mathrm{S} 2$, or -S3. The SCUBA-2 observations filtered out the large-scale $\left(>200^{\prime \prime}\right)$ extended emission and only picked up the dense condensations inside the cores. Some dense cores show very flattened and extended structure, thus their emissions are mostly filtered out in SCUBA-2 observations. In total, we identified 22 condensations with signal-to-noise ratios larger than 3 from SCUBA-2 images. According to panel (d) of Figure 3, the SCUBA-2-detected condensations are consistently smaller than those detected by Herschel. The total integrated fluxes of these condensations were calculated from 2D Gaussian fits. The masses were consequently obtained 
Table 4

Parameters of the Dense Cores Derived from SCUBA-2 $850 \mu \mathrm{m}$ Data

\begin{tabular}{|c|c|c|c|c|c|c|c|c|c|c|}
\hline Name & $\begin{array}{l}\text { Coordinate(J2000) } \\
\text { (R.A., Decl.) }\end{array}$ & $\begin{array}{l}\text { Total Flux } \\
\text { (mJy) }\end{array}$ & $\begin{array}{l}\text { Peak Flux Density } \\
\text { (mJy) }\end{array}$ & $\begin{array}{l}\text { Deconvolved Size } \\
\text { (“×”) }\end{array}$ & $\begin{array}{l}\text { P.A } A^{\mathrm{a}} \\
\left({ }^{\circ}\right)\end{array}$ & $\begin{array}{c}R(\text { SCUBA-2 })^{\mathrm{b}} \\
\quad\left(10^{-2} \mathrm{pc}\right)\end{array}$ & $\begin{array}{l}N_{\mathrm{H}_{2}}(\text { SCUBA-2) } \\
\quad\left(10^{21} \mathrm{~cm}^{-2}\right)\end{array}$ & $\begin{array}{c}n_{\mathrm{H}_{2}} \\
\left(10^{4} \mathrm{~cm}^{-3}\right)\end{array}$ & $\begin{array}{l}M_{\text {condensation }} \\
\quad\left(M_{\odot}\right)\end{array}$ & $\operatorname{Remark}^{\mathrm{c}}$ \\
\hline G168.13-16.39-S1 & $(4: 13: 48.350,+28: 12: 31.114)$ & $1096( \pm 140)$ & 80.40 & $72 \times 50$ & 20 & 4.10 & $5.63( \pm 0.65)$ & $2.23( \pm 0.26)$ & $0.44( \pm 0.05)$ & H1 \\
\hline G168.13-16.39-S2 & $(4: 13: 54.792,+28: 11: 33.400)$ & $66( \pm 20)$ & 71.60 & $14 \times 14$ & 0 & 0.95 & $5.39( \pm 1.14)$ & $9.20( \pm 1.95)$ & $0.02( \pm 0.01)$ & $\ldots$ \\
\hline G168.72-15.48-S1 & $(4: 18: 39.688,+28: 23: 24.070)$ & $4038( \pm 182)$ & 183.43 & $89 \times 51$ & -79 & 4.63 & $12.49( \pm 1.27)$ & $4.39( \pm 0.45)$ & $1.25( \pm 0.13)$ & $\mathrm{H} 2$ \\
\hline G168.72-15.48-S2 & $(4: 18: 39.116,+28: 21: 56.082)$ & $1229( \pm 139)$ & 75.57 & $116 \times 37$ & -77 & 4.50 & $4.16( \pm 0.44)$ & $1.50( \pm 0.16)$ & $0.40( \pm 0.04)$ & $\ldots$ \\
\hline G168.72-15.48-S3 & $(4: 18: 03.409,+28: 22: 52.354)$ & $1424( \pm 195)$ & 102.70 & $126 \times 35$ & 84 & 4.55 & $4.74( \pm 0.59)$ & $1.69( \pm 0.21)$ & $0.46( \pm 0.06)$ & $\cdots$ \\
\hline G169.76-16.15-S1 & $(4: 19: 42.596,+27: 13: 38.231)$ & $1166( \pm 128)$ & 424.45 & $23 \times 20$ & -74 & 1.48 & $34.88( \pm 3.22)$ & $38.20( \pm 3.53)$ & $0.40( \pm 0.03)$ & H5 \\
\hline G169.76-16.15-S2 & $(4: 19: 41.612,+27: 16: 08.184)$ & $177( \pm 37)$ & 144.54 & $14 \times 14$ & 0 & 0.95 & $12.50( \pm 1.83)$ & $21.36( \pm 3.14)$ & $0.05( \pm 0.01)$ & $\ldots$ \\
\hline G169.76-16.15-S3 & $(4: 19: 23.793,+27: 14: 54.189)$ & $1034( \pm 125)$ & 76.65 & $84 \times 37$ & -74 & 3.83 & $5.42( \pm 0.60)$ & $2.29( \pm 0.25)$ & $0.37( \pm 0.04)$ & $\mathrm{H} 3$ \\
\hline G170.00-16.14-S1 & $(4: 19: 58.676,+27: 09: 58.420)$ & $1346 \pm 174)$ & 396.04 & $26 \times 24$ & 58 & 1.74 & $34.05( \pm 4.53)$ & $31.69( \pm 4.22)$ & $0.48( \pm 0.06)$ & $\mathrm{H} 2$ \\
\hline G170.00-16.14-S2 & $(4: 19: 51.105,+27: 11: 27.498)$ & $632( \pm 110)$ & 81.79 & $59 \times 41$ & 58 & 3.36 & $4.29( \pm 0.65)$ & $2.07( \pm 0.31)$ & $0.23( \pm 0.03)$ & $\mathrm{H} 1$ \\
\hline G170.26-16.02-S1 & $(4: 21: 07.522,+27: 02: 31.323)$ & $257( \pm 67)$ & 56.54 & $31 \times 25$ & -49 & 1.93 & $5.01( \pm 1.09)$ & $4.22( \pm 0.92)$ & $0.09( \pm 0.02)$ & $\cdots$ \\
\hline G170.26-16.02-S2 & $(4: 21: 11.866,+27: 01: 13.907)$ & $578( \pm 81)$ & 83.59 & $49 \times 21$ & -54 & 2.19 & $8.74( \pm 1.20)$ & $6.47( \pm 0.88)$ & $0.20( \pm 0.03)$ & $\mathrm{H} 1$ \\
\hline G170.26-16.02-S3 & $(4: 21: 21.236,+26: 59: 53.873)$ & $798( \pm 96)$ & 70.25 & $70 \times 25$ & -54 & 2.91 & $7.03( \pm 0.90)$ & $3.92( \pm 0.50)$ & $0.28( \pm 0.04)$ & $\mathrm{H} 2$ \\
\hline G171.49-14.90-S1 & $(4: 28: 39.417,+26: 51: 37.576)$ & $2734( \pm 150)$ & 249.51 & $60 \times 45$ & -13 & 3.56 & $17.00( \pm 1.08)$ & $7.75( \pm 0.49)$ & $1.01( \pm 0.06)$ & $\mathrm{H} 1$ \\
\hline G171.49-14.90-S2 & $(4: 29: 04.907,+26: 49: 08.135)$ & $200( \pm 29)$ & 170.36 & $14 \times 14$ & 0 & 0.95 & $14.21( \pm 1.53)$ & $24.28( \pm 2.61)$ & $0.06( \pm 0.01)$ & $\cdots$ \\
\hline G171.80-15.32-S1 & $(4: 28: 10.207,+26: 20: 28.341)$ & $2667( \pm 155)$ & 186.27 & $91 \times 61$ & 63 & 5.07 & $7.73( \pm 0.60)$ & $2.47( \pm 0.19)$ & $0.93( \pm 0.07)$ & H1 \\
\hline G171.80-15.32-S2 & $(4: 27: 57.598,+26: 19: 25.435)$ & $1735( \pm 169)$ & 146.73 & $67 \times 46$ & 64 & 3.81 & $10.43( \pm 1.46)$ & $4.45( \pm 0.62)$ & $0.71( \pm 0.10)$ & $\mathrm{H} 2$ \\
\hline G171.80-15.32-S3 & $(4: 27: 49.314,+26: 18: 09.353)$ & $2871( \pm 155)$ & 65.58 & $136 \times 52$ & 63 & 5.76 & $7.15( \pm 0.78)$ & $2.02( \pm 0.22)$ & $1.11( \pm 0.12)$ & H3 \\
\hline G171.91-15.65-S1 & $(4: 27: 04.776,+26: 06: 18.335)$ & $858( \pm 21)$ & 88.37 & $14 \times 14$ & 0 & 0.95 & $67.36( \pm 2.33)$ & $115.07( \pm 3.97)$ & $0.29( \pm 0.01)$ & $\mathrm{H} 1$ \\
\hline G171.91-15.65-S2 & $(4: 27: 02.654,+26: 05: 32.941)$ & $1092( \pm 8.33)$ & 108.65 & $14 \times 14$ & 0 & 0.95 & $80.95( \pm 3.23)$ & $138.30( \pm 5.53)$ & $0.34( \pm 0.01)$ & $\mathrm{H} 2$ \\
\hline
\end{tabular}

Notes.

${ }^{\text {a }}$ Position angle of condensations detected by SCUBA-2; the convention used for measuring angles is east of north

$\mathrm{b}$ The radii are derived from elliptic Gaussian fitting to SCUBA-2 condensations

${ }^{c} \mathrm{H} 1, \mathrm{H} 2, \mathrm{H} 3$, or $\mathrm{H} 5$ represent the Herschel counterparts of condensations. 


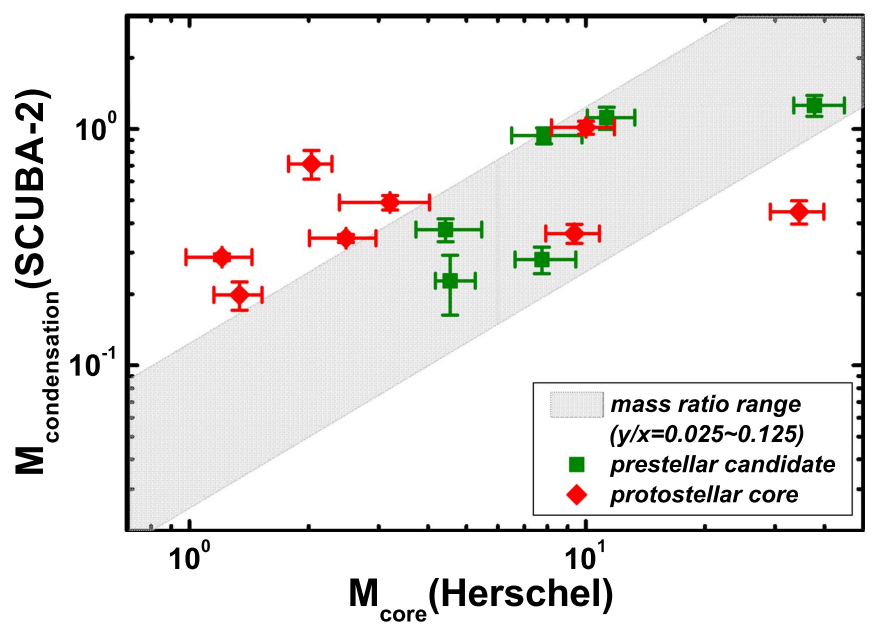

Figure 4. The correlation between $M_{\text {core }}$ (Herschel) and $M_{\text {condensation }}$ (SCUBA-2). The green squares and red diamonds represent prestellar candidates and protostellar cores, respectively. The gray area is the mass ratio range $(0.025 \sim 0.125)$. All prestellar candidates are covered by the gray area, but most protostellar cores have mass ratios higher than 0.125 .

using Kauffmann et al. (2008):

$$
\begin{aligned}
M= & 0.12 M_{\odot}\left(e^{1.439(\lambda / \mathrm{mm})^{-1}(T / 10 \mathrm{~K})^{-1}}-1\right) \\
& \times\left(\frac{\kappa_{\nu}}{0.01 \mathrm{~cm}^{2} \mathrm{~g}^{-1}}\right)^{-1}\left(\frac{S_{\nu}}{\mathrm{Jy}}\right)\left(\frac{D}{100 \mathrm{pc}}\right)^{2}\left(\frac{\lambda}{\mathrm{mm}}\right)^{3},
\end{aligned}
$$

where $T$ is temperature, adopting the Herschel dust temperature in our calculation, $D$ is the distance to the source, and $\kappa_{\nu}=0.012$ is the dust opacity at a $850 \mu \mathrm{m}$ wavelength, which is consistent with the value used in the SED fit to the Herschel data in Section 2.1. $S_{\nu}$ is the total flux density of the core region. The masses of the 22 SCUBA-2 condensations range from $0.02( \pm 0.01) M_{\odot}$ to $1.25( \pm 0.13) M_{\odot}$ and the column densities range from $4.2( \pm 0.5) \times 10^{21} \mathrm{~cm}^{-2}$ to $8.1( \pm 0.3) \times 10^{22} \mathrm{~cm}^{-2}$. The volume densities range from $1.5( \pm 0.2) \times 10^{4} \mathrm{~cm}^{-3}$ to $1.4( \pm 0.06) \times 10^{6} \mathrm{~cm}^{-3}$. All core parameters derived from SCUBA-2 data are presented in Table 4.

SCUBA-2 can detect condensations denser than those detected by Herschel. The Herschel volume densities of dense cores range from $5.7( \pm 0.9) \times 10^{3}$ to $7.9( \pm 1.4) \times 10^{4} \mathrm{~cm}^{-3}$. The volume densities derived from SCUBA-2 data range from $7.4( \pm 1.5) \times 10^{3}$ to $9.1( \pm 0.6) \times 10^{5} \mathrm{~cm}^{-3}$. The volume density estimated from the SCUBA-2 data is larger than the value estimated from the Herschel data, indicating that the SCUBA-2 detected condensations are denser than dense cores detected by Herschel. Figure 4 presents the correlation between $M_{\text {condensation }}(\mathrm{SCUBA}-2)$ and $M_{\text {core }}($ Herschel). The gray area represents mass ratios that range from 0.025 to 0.125 . The green squares and red diamonds represent prestellar candidates and protostellar cores, respectively. All prestellar candidates in Figure 4 are covered by a gray area, but most protostellar cores have mass ratios higher than 0.125 . This indicates that protostellar cores are generally more concentrated than prestellar candidates. This may indicate that as cores evolve, the volume density distributions of dense cores will be more centrally concentrated.

Ward-Thompson et al. (2016) identified 25 dense condensations in the L1495 cloud based on SCUBA-2 observations.
According to their results, the mass of dense condensations ranges from 0.02 to $0.61 M_{\odot}$, with a mean value of 0.19 . Their radii range from 0.02 to $0.03 \mathrm{pc}$, with a mean value of $0.03 \mathrm{pc}$. It should be noted that they have taken $\beta$ as 1.3 to the derived $\kappa_{\nu}$ in their calculations. If $\beta=2$ were taken, their condensations' masses would be doubled and be very similar to our SCUBA-2-detected condensations in PGCCs (mass ranging from 0.02 to $1.25 M_{\odot}$, with a mean value of $0.45 M_{\odot}$, and radii ranging from 0.02 to $0.06 \mathrm{pc}$, with a mean value of $0.03 \mathrm{pc}$.)

\subsection{Results of PMO Molecular Lines}

The ${ }^{12} \mathrm{CO}(1-0),{ }^{13} \mathrm{CO}(1-0)$, and $\mathrm{C}^{18} \mathrm{O}(1-0)$ lines were observed simultaneously with the PMO telescope. The coreaverage ${ }^{12} \mathrm{CO}(1-0),{ }^{13} \mathrm{CO}(1-0)$, and $\mathrm{C}^{18} \mathrm{O}(1-0)$ spectra of 30 Herschel dense cores are presented in Figure 5. The spectra of ${ }^{12} \mathrm{CO}(1-0),{ }^{13} \mathrm{CO}(1-0)$, and $\mathrm{C}^{18} \mathrm{O}(1-0)$ are shown in red, green, and blue, respectively. From Gaussian fitting, we obtain peak velocity, full width of half maximum (FWHM), and peak brightness temperature. Some dense cores (17 dense cores) show multiple velocity components; for these we fitted multiple Gaussian components. The fitting parameters of all dense cores are presented in Table 5. For lines with multiple velocity components, the velocity component is determined to be associated with the Herschel dense core if its integrated intensity map shows a similar morphology to its Herschel map.

The excitation temperature $T_{\mathrm{ex}}$ of ${ }^{12} \mathrm{CO}$ is calculated following (Garden et al. 1991)

$$
\begin{aligned}
T_{\mathrm{b}}= & \frac{T_{\mathrm{a}}^{*}}{\eta_{b}}=\frac{h \nu}{k}\left[\frac{1}{e^{\left(h \nu / k T_{\mathrm{ex}}\right)}-1}-\frac{1}{e^{\left(h \nu / k T_{\mathrm{bg}}\right)}-1}\right] \\
& \times\left(1-e^{-\tau}\right) f,
\end{aligned}
$$

where $T_{\mathrm{b}}$ is the brightness temperature. $T_{\mathrm{bg}}$ is the background temperature of $2.73 \mathrm{~K} . T_{\mathrm{a}}^{*}$ is the observed antenna temperature, $k$ is the Boltzmann constant, $\eta_{\mathrm{b}}=0.6$ is the main beam efficiency, and $\nu$ is the frequency. Assuming that ${ }^{12} \mathrm{CO}(1-0)$ is optically thick $(\tau \gg 1)$ and that the filling factor $f=1$, then $T_{\text {ex }}$ can be obtained. The mean, maximum, minimum, and median $T_{\text {ex }}$ values of each dense core are presented in Table 6. The mean $T_{\mathrm{ex}}$ ranges from 8.5 to $15.1 \mathrm{~K}$, consistent with the dust temperature range.

If we assume that ${ }^{12} \mathrm{CO}(1-0)$ and ${ }^{13} \mathrm{CO}(1-0)$ have the same excitation temperature, the ${ }^{13} \mathrm{CO}$ optical depth can be obtained. These values are also presented in Table 6 . Then, the ${ }^{13} \mathrm{CO}$ column density can be calculated as follows (Garden et al. 1991):

$$
N=\frac{3 k}{8 \pi^{2} B \mu_{\mathrm{D}}^{2}} \frac{e^{\left[h B J(J+1) / k T_{\mathrm{ex}}\right]}}{J+1} \times \frac{T_{\mathrm{ex}}+h B / 3 k}{1-e^{\left(-h \nu / k T_{\mathrm{ex}}\right)}} \int \tau_{v} d v,
$$

where $B=55101.012 \mathrm{MHz}$ is the rotational constant, $\mu_{\mathrm{D}}=0.11$ debyes is the permanent dipole moment for ${ }^{13} \mathrm{CO}$, and $J$ is the rotational quantum number of the lower state in the observed transition (Chackerian \& Tipping 1983). In this paper, we adopt a typical abundance ratio of $\left[{ }^{12} \mathrm{CO}\right] /\left[{ }^{13} \mathrm{CO}\right]=60$ and $\left[\mathrm{H}_{2}\right] /\left[{ }^{12} \mathrm{CO}\right]=10^{4}$, considering the $\left[{ }^{12} \mathrm{CO}\right] /\left[{ }^{13} \mathrm{CO}\right]$ ratio of $\sim 50$ shown by Hawkins \& Jura (1987) for the solar neighborhood and the ratio of $\sim 70$ in the Galaxy shown by Penzias (1980). With these assumptions, the $\mathrm{H}_{2}$ column densities are calculated and also presented in Table 6. The mean, maximum, minimum, and median values of the column 


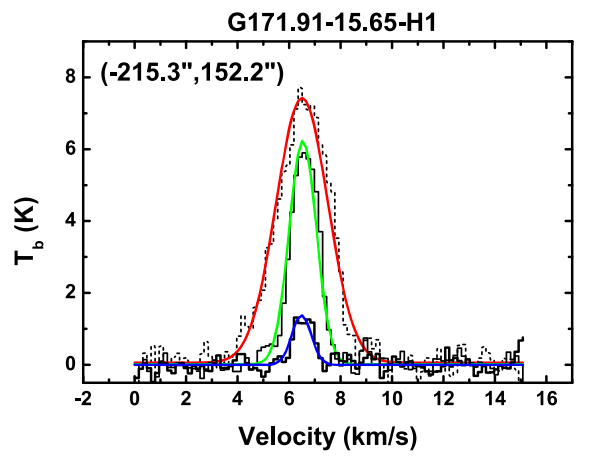

(a)

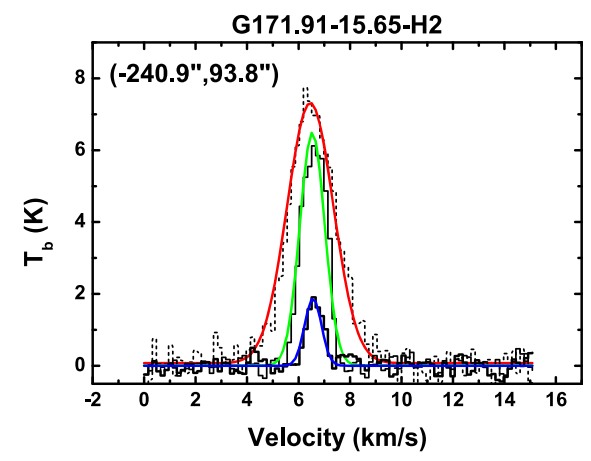

(b)

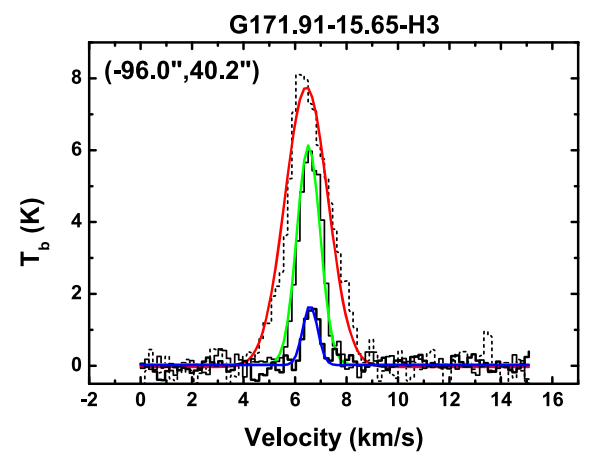

(c)

Figure 5. Average spectra of three dense cores for the representative source PGCC G171.91-15.65. The images for other sources are shown in the figure set. For each plot, the three lines of ${ }^{12} \mathrm{CO},{ }^{13} \mathrm{CO}$, and $\mathrm{C}^{18} \mathrm{O}$ are in red, green, and blue, respectively. The offsets of dense cores are presented in the upper left corner in units of arcseconds, and they are measured relative to coordinates of PGCCs released by Planck Collaboration et al. (2016). The core names are provided above each panel. The images for other sources are shown in the figure set.

(The complete figure set (30 images) is available.)

density of each dense core are also given in columns 2 through 5 , respectively. The $N_{\mathrm{H}_{2}}$ distributions, derived from PMO data, are presented in panel (b) of Figure 3.

The thermal velocity dispersion $\left(\sigma_{\text {th }}\right)$ and non-thermal velocity dispersion $\left(\sigma_{\mathrm{NT}}\right)$ are calculated as

$$
\begin{gathered}
\sigma_{\mathrm{NT}}=\left[\sigma_{{ }^{23} \mathrm{CO}}-\frac{k T_{\mathrm{ex}}}{m{ }^{13} \mathrm{CO}}\right]^{1 / 2}, \\
\sigma_{\mathrm{th}}=\sqrt{\frac{k T_{\mathrm{ex}}}{m_{\mathrm{H}} \mu}},
\end{gathered}
$$

where $m^{13} \mathrm{CO}$ is the mass of the ${ }^{13} \mathrm{CO}$ molecule, $m_{\mathrm{H}}$ is the atomic hydrogen mass, and $\mu=2.8$ is the mean molecular weight of the gas (Kauffmann et al. 2008). $\sigma^{{ }^{13} \mathrm{CO}}$ is the $1 \mathrm{D}$ velocity dispersion, obtained from second-moment maps. If the velocity dispersion is isotropic, the 3D velocity dispersions are calculated using

$$
\sigma_{3 \mathrm{D}}=\sqrt{3\left(\sigma_{\mathrm{th}}^{2}+\sigma_{\mathrm{NT}}^{2}\right)} .
$$

The mean, maximum, minimum, and median $\sigma_{\mathrm{NT}}, \sigma_{\mathrm{th}}$, and $\sigma_{3 \mathrm{D}}$ values of each dense core are also presented in Table 6. The mean values of $\sigma_{\mathrm{NT}}$, derived from ${ }^{13} \mathrm{CO}$, range from $0.36( \pm 0.09) \mathrm{km} \mathrm{s}^{-1}$ to $0.73( \pm 0.07) \mathrm{km} \mathrm{s}^{-1}$. The mean values of $\sigma_{\text {th }}$ range from $0.16( \pm 0.01)$ to $0.21( \pm 0.01) \mathrm{km} \mathrm{s}^{-1}$.

If ${ }^{13} \mathrm{CO}$ is optically thick, $\sigma_{\mathrm{NT}}$ could be overestimated. Actually, ${ }^{13} \mathrm{CO}$ is optically thick in PGCCs (Wu et al. 2012). The high spectral resolution $\left(0.16 \mathrm{~km} \mathrm{~s}^{-1}\right)$ in the PMO observations can also resolve the $\mathrm{C}^{18} \mathrm{O}$ line profiles. Therefore, we also calculated $\sigma_{\mathrm{NT}}$ using $\mathrm{C}^{18} \mathrm{O}$ data, and the $\sigma_{\mathrm{NT}}\left(\mathrm{C}^{18} \mathrm{O}\right)$ values are presented in Table 8 . The $\sigma_{\mathrm{NT}}\left(\mathrm{C}^{18} \mathrm{O}\right)$ values range from $0.19( \pm 0.01) \mathrm{km} \mathrm{s}^{-1}$ to $0.71( \pm 0.21) \mathrm{km} \mathrm{s}^{-1}$. The Mach number $\left(\sigma_{\mathrm{NT}}\left(\mathrm{C}^{18} \mathrm{O}\right) / \sigma_{\mathrm{th}}\right)$ can be calculated and listed in Table 8, where $\sigma_{\text {th }}$ is the isothermal sound speed. The Mach number of dense cores ranges from $1( \pm 0.08)$ to $3.8( \pm 1.2)$, with a mean value of $2.1( \pm 0.8)$. Thus, we conclude that all the dense cores studied here have supersonic non-thermal motions.

For all of the dense cores, the Mach numbers are always greater than 1, and there is not much difference in Mach number among starless cores, prestellar core candidates, and protostellar cores. The large Mach numbers also indicate that the dense cores may be turbulence-dominated. The non-thermal velocity dispersions, however, could also be caused by bulk motions like infall or rotation.

The integrated intensity maps of $\mathrm{C}^{18} \mathrm{O}(1-0)$ are shown in panel (c) of Figure 3. The $\mathrm{C}^{18} \mathrm{O}$ map is very noisy (the average signal-to-noise ratio of the $\mathrm{C}^{18} \mathrm{O}$ mapping region is $4.1 \pm 1.2$ ). Therefore, it is hard to derive accurate $\mathrm{C}^{18} \mathrm{O}$ column density maps. Instead, we calculate core-averaged $\mathrm{C}^{18} \mathrm{O}$ column densities from the core-averaged $\mathrm{C}^{18} \mathrm{O}$ spectra.

If we assume that $\mathrm{C}^{18} \mathrm{O}$ emission is optically thin and the excitation temperature is the same as the Herschel dust temperature $\left(T_{\mathrm{d}}\right)$, the $N_{\mathrm{C}^{18} \mathrm{O}}$ (column density of $\mathrm{C}^{18} \mathrm{O}$ ) is calculated from Garden et al. (1991) as

$$
N_{\mathrm{C}^{18} \mathrm{O}}=4.77 \times 10^{13} \frac{\left(T_{\mathrm{ex}}+0.89\right)}{e^{\left(-5.27 / T_{\mathrm{ex}}\right)}} \int \frac{T_{\mathrm{a}}^{*}\left(\mathrm{C}^{18} \mathrm{O}\right)}{\eta_{\mathrm{b}}} d v,
$$

where $T_{\mathrm{a}}^{*}$ is antenna temperature, and $\eta_{\mathrm{b}}=0.6$ is the main beam efficiency of the PMO telescope.

We applied the non-LTE code "Radex" (Van der Tak et al. 2007) to fit $\mathrm{C}^{18} \mathrm{O}$ parameters. The input parameters for Radex are volume density, kinetic temperature, and line width. We used volume density from Herschel observations and assumed that the kinetic temperature equals the dust temperature. The line widths were obtained from Gaussian fits to the PMO $\mathrm{C}^{18} \mathrm{O}(1-0)$ spectra. The derived column density, excitation temperature, and optical depth from Radex, and the LTE column density derived from PMO data, are summarized in Table 7.

Figure 6 shows the correlation between the LTE $\mathrm{C}^{18} \mathrm{O}$ column density and the non-LTE $\mathrm{C}^{18} \mathrm{O}$ column density. The correlation is well fitted by a power-law function, and the fitted function is close to $N_{\mathrm{C}^{18} \mathrm{O}}(\mathrm{LTE})=N_{\mathrm{C}^{18} \mathrm{O}}$ (non-LTE), which indicates that both the calculations are reasonable.

\section{Discussion \\ 4.1. Stabilities Of Dense Cores}

The gravitational stability is a critical factor for determining whether a dense core is gravitationally bound or not. Assuming a Gaussian velocity distribution and uniform density distribution, the dense core may be supported solely by random motions. For this configuration, a virial mass can be estimated 
Table 5

The Parameters Derived from Spectra of Dense Cores

\begin{tabular}{|c|c|c|c|c|c|c|c|c|c|}
\hline Name & $\begin{array}{l}V_{\mathrm{lsr}}(12) \\
\left(\mathrm{km} \mathrm{s}^{-1}\right)\end{array}$ & $\begin{array}{c}\mathrm{FWHM}(12) \\
\left(\mathrm{km} \mathrm{s}^{-1}\right)\end{array}$ & $\begin{array}{l}T_{\mathrm{b}}(12) \\
\quad(\mathrm{K})\end{array}$ & $\begin{array}{l}V_{\mathrm{lsr}}(13) \\
\left(\mathrm{km} \mathrm{s}^{-1}\right)\end{array}$ & $\begin{array}{c}\mathrm{FWHM(13)} \\
\left(\mathrm{km} \mathrm{s}^{-1}\right)\end{array}$ & $\begin{array}{c}T_{\mathrm{b}}(13) \\
(\mathrm{K})\end{array}$ & $\begin{array}{l}V_{\mathrm{lsr}}(18) \\
\left(\mathrm{km} \mathrm{s}^{-1}\right)\end{array}$ & $\begin{array}{c}\mathrm{FWHM}(18) \\
\left(\mathrm{km} \mathrm{s}^{-1}\right)\end{array}$ & $\begin{array}{l}T_{\mathrm{b}}(18) \\
(\mathrm{K})\end{array}$ \\
\hline \multirow[t]{2}{*}{ G166.99-15.34-H1 } & $8.01( \pm 0.01)$ & $0.98( \pm 0.06)$ & $1.8( \pm 0.5)$ & $5.65( \pm 0.04)$ & $2.60( \pm 0.26)$ & 0.3 & & & \\
\hline & $6.38( \pm 0.02)$ & $2.98( \pm 0.04)$ & $4.7( \pm 0.3)$ & & & & $6.93( \pm 0.10)$ & $1.00( \pm 0.38)$ & $.2( \pm 0.1)$ \\
\hline \multirow[t]{2}{*}{ G167.23-15.32-H1 } & $4.42( \pm 0.04)$ & $1.23( \pm 0.12)$ & $3.1( \pm 0.6)$ & $4.23( \pm 0.24)$ & $2.05( \pm 0.53)$ & $0.4( \pm 0.3)$ & & & \\
\hline & $6.92( \pm 0.04)$ & $2.97( \pm 0.10)$ & $5.8( \pm 0.4)$ & $6.58( \pm 0.03)$ & $1.54( \pm 0.08)$ & $2.8( \pm 0.3)$ & $6.65( \pm 0.04)$ & $0.53( \pm 0.13)$ & $1.0( \pm 0.3)$ \\
\hline \multirow[t]{2}{*}{ G168.00-15.69-H1 } & $4.93( \pm 0.03)$ & $1.22( \pm 0.05)$ & $1.5( \pm 0.8)$ & & & & & & \\
\hline & $7.95( \pm 0.01)$ & $1.81( \pm 0.01)$ & $10.1( \pm 0.7)$ & $7.83( \pm 0.01)$ & $0.92( \pm 0.01)$ & $6.4( \pm 0.2)$ & $7.79( \pm 0.01)$ & $0.55( \pm 0.02)$ & $1.8( \pm 0.1)$ \\
\hline \multirow[t]{2}{*}{ G168.13-16.39-H1 } & $6.22( \pm 0.01)$ & $1.26( \pm 0.02)$ & $8.3( \pm 0.7)$ & $6.23( \pm 0.01)$ & $0.61( \pm 0.01)$ & $3.9( \pm 0.4)$ & $6.60( \pm 0.01)$ & $0.75( \pm 0.02)$ & $2.8( \pm 0.2)$ \\
\hline & $7.46( \pm 0.01)$ & $0.92( \pm 0.02)$ & $7.2( \pm 0.7)$ & $7.00( \pm 0.01)$ & $0.90( \pm 0.01)$ & $5.5( \pm 0.3)$ & & & \\
\hline \multirow[t]{2}{*}{ G168.13-16.39-H2 } & $3.60( \pm 0.06)$ & $1.57( \pm 0.13)$ & $2.0( \pm 0.6)$ & & & & & & \\
\hline & $6.81( \pm 0.02)$ & $2.09( \pm 0.04)$ & $8.6( \pm 0.5)$ & $6.84( \pm 0.01)$ & $1.24( \pm 0.01)$ & $6.6( \pm 0.3)$ & $6.84( \pm 0.02)$ & $0.88( \pm 0.04)$ & $2.1( \pm 0.2)$ \\
\hline G168.72-15.48-H1 & $7.34( \pm 0.02)$ & $3.04( \pm 0.04)$ & $9.7( \pm 0.5)$ & $7.34( \pm 0.02)$ & $1.28( \pm 0.04)$ & $6.5( \pm 0.5)$ & $7.37( \pm 0.02)$ & $0.57( \pm 0.04)$ & $2.7( \pm 0.4)$ \\
\hline G168.72-15.48-H2 & $7.40( \pm 0.01)$ & $2.74( \pm 0.03)$ & $10.3( \pm 0.4)$ & $7.32( \pm 0.01)$ & $1.27( \pm 0.02)$ & $7.1( \pm 0.4)$ & $7.31( \pm 0.01)$ & $0.68( \pm 0.03)$ & $3.9( \pm 0.3)$ \\
\hline \multirow[t]{2}{*}{ G169.43-16.17-H1 } & $5.24( \pm 0.02)$ & $1.80( \pm 0.04)$ & & $5.35( \pm 0.02)$ & & $5.0( \pm$ & $5.53( \pm 0.03)$ & $1.30( \pm 0.07)$ & $1.4( \pm 0.2)$ \\
\hline & $7.76( \pm 0$ & $1.92( \pm$ & & $6.93( \pm 0$ & & & & & \\
\hline \multirow[t]{2}{*}{ G169.43-16.17-H2 } & $4.96( \pm 0.02)$ & $1.89( \pm 0.05)$ & $7.8( \pm 0.6)$ & $5.37( \pm 0.01)$ & $1.65( \pm 0.03)$ & $5.6( \pm 0.3)$ & $5.64( \pm 0.03)$ & $0.98( \pm 0.09)$ & $1.6( \pm 0.2)$ \\
\hline & $7.56( \pm 0.02)$ & 1.63 & $7.9( \pm 0.7)$ & 7.52( & 1.29 & 2.0( & & & \\
\hline \multirow[t]{2}{*}{ G169.76-16.15-H1 } & $5.21( \pm 0.16)$ & 1.67( & $7.1( \pm 0.9)$ & $5.52( \pm 0.01)$ & $1.76( \pm 0.03)$ & $5.2( \pm 0.4)$ & $5.69( \pm 0.02)$ & $1.28( \pm 0.06)$ & $1.9( \pm 0.2)$ \\
\hline & $7.59( \pm 0.16)$ & $1.75( \pm 0.16)$ & $9.3( \pm 0.9)$ & $=0.03)$ & $1.19( \pm 0.08)$ & 1.8( & & & \\
\hline \multirow[t]{2}{*}{ G169.76-16.15-H2 } & $5.32( \pm 0.01)$ & $1.39( \pm 0.02)$ & $7.3( \pm 0.6)$ & $5.42( \pm 0.01)$ & $1.05( \pm 0.02)$ & $4.4( \pm 0.8)$ & $5.83( \pm 0.02)$ & $1.15( \pm 0.05)$ & $2.0( \pm 0.2)$ \\
\hline & $7.56( \pm 0.01)$ & $1.94( \pm 0.03)$ & $9.2( \pm 0.6)$ & $6.71( \pm 0.03)$ & $2.18( \pm 0.05)$ & $3.4( \pm 0.6)$ & & & \\
\hline \multirow[t]{2}{*}{ G169.76-16.15-H3 } & $5.40( \pm 0.01)$ & $1.39( \pm 0.01)$ & $7.6( \pm 0.6)$ & $5.40( \pm 0.01)$ & $0.88( \pm 0.02)$ & $4.5( \pm 0.5)$ & $5.52( \pm 0.01)$ & $0.59( \pm 0.02)$ & $2.2( \pm 0.2)$ \\
\hline & $7.49( \pm 0.01)$ & $1.61( \pm 0.01)$ & $9.2( \pm 0.6)$ & $6.84( \pm 0.02)$ & $1.88( \pm 0.04)$ & $3.7(t$ & 6.90( & 0.72 & $1.2( \pm 0.1)$ \\
\hline \multirow[t]{2}{*}{ G169.76-16.15-H4 } & $5.48( \pm 0.01)$ & $1.38( \pm 0.02)$ & $7.6( \pm 0.5)$ & $5.45( \pm 0.01)$ & $0.67( \pm 0.02)$ & $2.8( \pm$ & $5.59( \pm 0.03)$ & $0.58( \pm 0.08)$ & $0.6( \pm 0.2)$ \\
\hline & $7.58( \pm 0.01)$ & $1.51( \pm 0.02)$ & $8.3( \pm 0.5)$ & $6.83( \pm 0.01)$ & $1.64( \pm 0.03)$ & $3.9( \pm 0.3)$ & $6.88( \pm 0.01)$ & $0.60( \pm 0.03)$ & $1.6( \pm 0.2)$ \\
\hline \multirow[t]{2}{*}{ G169.76-16.15-H5 } & $5.57( \pm 0.01)$ & $1.28( \pm 0.03)$ & $7.8( \pm 0.6)$ & $5.59( \pm 0.02)$ & $0.77( \pm 0.03)$ & $3.8( \pm$ & $5.71( \pm 0.06)$ & $0.89( \pm 0.13)$ & $0.6( \pm 0.2)$ \\
\hline & $7.57( \pm 0.02)$ & & & & & & & $0.51( \pm 0.05)$ & $1.3( \pm 0.2)$ \\
\hline \multirow{2}{*}{ G170.00-16.14-H1 } & 5.47 & & & & & & & & \\
\hline & $7.36( \pm 0.03)$ & & 5. & 6. & 11) & 3.6 & $6.59( \pm 0.08)$ & $1.11( \pm 0.13)$ & $0.7( \pm 0.3)$ \\
\hline \multirow[t]{2}{*}{ G170.00-16.14-H2 } & $5.30( \pm 0.02)$ & $1.30( \pm 0.04)$ & $7.5( \pm 0.6)$ & $5.31( \pm 0.05)$ & $0.88( \pm 0.11)$ & $1.8( \pm 0.4)$ & & & \\
\hline & $7.40( \pm 0.04)$ & $2.12( \pm 0.09)$ & $5.2( \pm 0.5)$ & $6.64( \pm 0.03)$ & $1.56( \pm 0.08)$ & $4.4( \pm 0.4)$ & $6.61( \pm 0.05)$ & $1.11( \pm 0.10)$ & $0.9( \pm 0.2)$ \\
\hline \multirow[t]{2}{*}{ G170.13-16.06-H1 } & $5.94( \pm 0.02)$ & $1.48( \pm 0.04)$ & $7.2( \pm 0.5)$ & $6.00( \pm 0.02)$ & $0.96( \pm 0.03)$ & $4.5( \pm 0.4)$ & $5.97( \pm 0.06)$ & $0.62( \pm 0.17)$ & $0.6( \pm 0.3)$ \\
\hline & $7.38( \pm 0.02)$ & $1.00( \pm 0.04)$ & $4.2( \pm 0.6)$ & $6.96( \pm 0.02)$ & $0.91( \pm 0.03)$ & $4.1( \pm 0.4)$ & $6.85( \pm 0.03)$ & $0.80( \pm 0.07)$ & $1.4( \pm 0.3)$ \\
\hline G170.26-16.02-H1 & $6.23( \pm 0.02)$ & $2.36( \pm 0.04)$ & $6.7( \pm 0.7)$ & $6.36( \pm 0.01)$ & $1.66( \pm 0.02)$ & $5.1( \pm 0.4)$ & $6.64( \pm 0.04)$ & $1.41( \pm 0.09)$ & $1.1( \pm 0.2)$ \\
\hline \multirow[t]{2}{*}{ G170.26-16.02-H2 } & $5.99( \pm 0.05)$ & $1.22( \pm 0.08)$ & $6.7( \pm 1.2)$ & $6.51( \pm 0.01)$ & $1.24( \pm 0.01)$ & $5.5( \pm 0.2)$ & $6.56( \pm 0.01)$ & $0.83( \pm 0.04)$ & $1.6( \pm 0.1)$ \\
\hline & $7.17( \pm 0.06)$ & $1.13( \pm 0.09)$ & $4.9( \pm 1.2)$ & & & & & & \\
\hline G170.83-15.90-H1 & $6.61( \pm 0.02)$ & $1.46( \pm 0.04)$ & $6.2( \pm 0.4)$ & $6.53( \pm 0.01)$ & $1.06( \pm 0.02)$ & & & & $1.3( \pm 0.3)$ \\
\hline G170.83-15.90-H2 & $6.67( \pm 0.02)$ & $1.73( \pm 0.04)$ & $6.2( \pm 0.5)$ & $6.66( \pm 0.01)$ & $1.18( \pm 0.02)$ & $5.0( \pm 0.2)$ & $6.79( \pm 0.07)$ & $0.95( \pm 0.17)$ & $1.1( \pm 0.3)$ \\
\hline \multirow[t]{3}{*}{ G170.99-15.81-H1 } & & & & & & & & & \\
\hline & 6.78 & & $4.3( \pm 0$ & $6.50( \pm 0.01)$ & $1.16( \pm 0.03)$ & $4.8( \pm 0.2)$ & $6.51( \pm 0.02)$ & $0.64( \pm 0.05)$ & $2.2( \pm 0.3)$ \\
\hline & $8.07( \pm 0.07)$ & $0.71( \pm 0.17)$ & $1.7( \pm 0.8)$ & & & & & & \\
\hline \multirow[t]{2}{*}{ G171.49-14.90-H1 } & $5.95( \pm 0.16)$ & $1.29( \pm 0.16)$ & $7.0( \pm 0.6)$ & $6.52( \pm 0.02)$ & $0.92( \pm 0.04)$ & $5.3( \pm 0.5)$ & $6.55( \pm 0.02)$ & $0.47( \pm 0.04)$ & $2.4( \pm 0.4)$ \\
\hline & $7.49( \pm 0.16)$ & $1.31( \pm 0.16)$ & $8.4( \pm 0.7)$ & $7.58( \pm 0.03)$ & $0.85( \pm 0.05)$ & $3.2( \pm 0.5)$ & & & \\
\hline G171.80-15.32-H1 & $6.90( \pm 0.26)$ & $1.77( \pm 0.04)$ & $7.4( \pm 0.5)$ & $6.99( \pm 0.01)$ & $0.96( \pm 0.02)$ & $5.7( \pm 0.3)$ & $6.96( \pm 0.06)$ & $1.22( \pm 0.13)$ & $0.9( \pm 0.2)$ \\
\hline G171.80-15.32-H2 & $9.06( \pm 0.02)$ & $2.17( \pm 0.05)$ & $7.4( \pm 0.5)$ & $7.23( \pm 0.02)$ & $1.29( \pm 0.04)$ & $5.2( \pm 0.3)$ & $7.38( \pm 0.13)$ & $1.67( \pm 0.30)$ & $0.7( \pm 0.3)$ \\
\hline G171.80-15.32-H3 & $7.04( \pm 0.02)$ & $1.96( \pm 0.04)$ & $6.7( \pm 0.4)$ & $7.17( \pm 0.01)$ & $1.01( \pm 0.02)$ & $5.1( \pm 0.3)$ & $7.12( \pm 0.11)$ & $1.44( \pm 0.27)$ & $0.5( \pm 0.3)$ \\
\hline G171.91-15.65-H1 & $6.59( \pm 0.02)$ & $2.36( \pm 0.04)$ & $7.4( \pm 0.4)$ & $6.61( \pm 0.01)$ & $1.25( \pm 0.03)$ & $6.2( \pm 0.3)$ & $6.56( \pm 0.04)$ & $0.90( \pm 0.09)$ & $1.4( \pm 0.3)$ \\
\hline G171.91-15.65-H2 & $6.54( \pm 0.02)$ & $2.12( \pm 0.05)$ & $7.3( \pm 0.4)$ & $6.61( \pm 0.01)$ & $1.14( \pm 0.02)$ & $6.5( \pm 0.3)$ & $6.64( \pm 0.03)$ & $0.78( \pm 0.06)$ & $1.9( \pm 0.3)$ \\
\hline G171.91-15.65-H3 & $6.52( \pm 0.02)$ & $1.97( \pm 0.05)$ & $7.8( \pm 0.4)$ & $6.60( \pm 0.01)$ & $1.06( \pm 0.02)$ & $6.1( \pm 0.3)$ & $6.66( \pm 0.02)$ & $0.69( \pm 0.06)$ & $1.7( \pm 0.3)$ \\
\hline G172.06-15.21-H1 & $6.59( \pm 0.01)$ & $1.72( \pm 0.02)$ & $9.3( \pm 0.2)$ & $6.77( \pm 0.01)$ & $1.01( \pm 0.02)$ & $5.1( \pm 0.2)$ & $6.81( \pm 0.01)$ & $0.57( \pm 0.03)$ & $1.8( \pm 0.2)$ \\
\hline
\end{tabular}

Note. All parameters are derived from spectra of dense cores, which averaged over the identified cores' regions. For some dense cores, there are multiple velocity components, but there are some velocity components that do not have ${ }^{13} \mathrm{CO}$ and $\mathrm{C}^{18} \mathrm{O}$ emission. The spectral plot can be seen in Figure 5 .

using (MacLaren et al. 1988)

$$
M_{\mathrm{vir}}=2.10 \times 10^{2}\left(\frac{R}{\mathrm{pc}}\right)\left(\frac{\Delta V}{\mathrm{~km} \mathrm{~s}^{-1}}\right)^{2} M_{\odot},
$$

where $\Delta V$ is the FWHM of $\mathrm{C}^{18} \mathrm{O}$ spectrum, which is derived from averaged $\mathrm{C}^{18} \mathrm{O}$ spectra over the Herschel dense core, and $R$ is the radius of the Herschel dense core. The $M_{\text {vir }}$ values of
30 cores range from $3.6( \pm 0.7) M_{\odot}$ to $74( \pm 7.6) M_{\odot} \quad$ (see Table 8).

We also calculated the virial parameter $\alpha=M_{\text {vir }} / M_{\text {core }}$. Kauffmann et al. (2013) and Friesen et al. (2016) consider $\alpha=2$ as a lower limit for gas motions to prevent collapse, unless the dense cores are supported by a significant magnetic field or confined by external pressure. The virial parameter of the dense cores analyzed here ranges from $0.3( \pm 0.1)$ to 17.5 $( \pm 7.3)$. Fourteen dense cores have virial parameters smaller 
Table 6

The Parameters of a Dense Core Derived from CO Gas Emission

\begin{tabular}{|c|c|c|c|c|c|c|c|c|c|c|c|c|c|c|c|c|c|c|c|c|c|}
\hline \multirow{2}{*}{ Name } & \multicolumn{4}{|c|}{$N_{\mathrm{H}_{2}}(\mathrm{PMO})$} & \multicolumn{4}{|c|}{$T_{\mathrm{ex}}$} & \multirow[t]{2}{*}{$\tau\left({ }^{13} \mathrm{CO}\right)$} & \multicolumn{4}{|c|}{$\sigma_{\text {th }}$} & \multicolumn{4}{|c|}{$\sigma_{\mathrm{NT}}\left({ }^{13} \mathrm{CO}\right)$} & \multicolumn{4}{|c|}{$\sigma_{3 \mathrm{D}}\left({ }^{13} \mathrm{CO}\right)$} \\
\hline & mean & $\begin{array}{c}\max \\
\left(10^{21} \mathrm{~cm}\right.\end{array}$ & $\min _{-2}$ & $\overline{\text { median }}$ & mean & $\begin{array}{l}\max \\
(\mathrm{K})\end{array}$ & $\min$ & $\overline{\text { median }}$ & & mean & $\begin{array}{l}\max \\
\left(\mathrm{km} \mathrm{s}^{-1}\right)\end{array}$ & $\min$ & median & mean & $\begin{array}{l}\max \\
\left(\mathrm{km} \mathrm{s}^{-1}\right.\end{array}$ & $\min$ & median & mean & $\begin{array}{l}\max \\
\left(\mathrm{km} \mathrm{s}^{-1}\right.\end{array}$ & $\min$ & median \\
\hline $\begin{array}{l}\text { G166.99- } \\
15.34-\mathrm{H} 1\end{array}$ & $2.1( \pm 0.2)$ & 2.4 & 1.7 & 2.1 & $8.5( \pm 0.3)$ & 9.0 & 7.8 & 8.4 & $0.34( \pm 0.03)$ & $0.15( \pm 0.003)$ & 0.16 & 0.14 & 0.15 & $0.56( \pm 0.11)$ & 0.83 & 0.22 & 0.62 & $1.02( \pm 0.29)$ & 1.45 & 0.45 & 1.11 \\
\hline $\begin{array}{l}\text { G167.23- } \\
15.32-\mathrm{H} 1\end{array}$ & $3.0( \pm 0.4)$ & 3.7 & 2.2 & 2.9 & $10.7( \pm 0.8)$ & 12.5 & 9.2 & 10.8 & $0.48( \pm 0.07)$ & $0.16( \pm 0.007)$ & 0.17 & 0.15 & 0.16 & $0.65( \pm 0.21)$ & 1.26 & 0.60 & 0.96 & $1.17( \pm 0.34)$ & 2.20 & 1.07 & 1.69 \\
\hline $\begin{array}{l}\text { G168.00- } \\
15.69-\mathrm{H} 1\end{array}$ & $4.8( \pm 0.3)$ & 5.5 & 3.9 & 4.8 & $15.1( \pm 0.7)$ & 16.8 & 13.9 & 14.9 & $0.78( \pm 0.07)$ & $0.21( \pm 0.005)$ & 0.23 & 0.21 & 0.21 & $0.48( \pm 0.06)$ & 0.62 & 0.38 & 0.49 & $0.91( \pm 0.09)$ & 1.14 & 0.75 & 0.92 \\
\hline $\begin{array}{l}\text { G168.13- } \\
16.39-\mathrm{H} 1\end{array}$ & $3.2( \pm 0.3)$ & 4.1 & 3.0 & 3.5 & $12.1( \pm 0.4)$ & 13.4 & 11.3 & 12.0 & $0.58( \pm 0.04)$ & $0.19( \pm 0.004)$ & 0.20 & 0.19 & 0.19 & $0.37( \pm 0.04)$ & 0.44 & 0.27 & 0.38 & $0.73( \pm 0.06)$ & 0.83 & 0.58 & 0.73 \\
\hline $\begin{array}{l}\text { G168.13- } \\
16.39-\mathrm{H} 2\end{array}$ & $3.5( \pm 0.3)$ & 4.1 & 3.1 & 3.5 & $12.5( \pm 0.7)$ & 14.1 & 11.4 & 12.3 & $1.30( \pm 0.20)$ & $0.19( \pm 0.005)$ & 0.21 & 0.19 & 0.19 & $0.36( \pm 0.06)$ & 0.48 & 0.26 & 0.37 & $0.70( \pm 0.09)$ & 0.91 & 0.55 & 0.72 \\
\hline $\begin{array}{l}\text { G168.72- } \\
15.48-\mathrm{H} 1\end{array}$ & $6.8( \pm 0.5)$ & 8.1 & 5.6 & 6.9 & $14.4( \pm 0.4)$ & 16.6 & 12.3 & 14.3 & $1.03( \pm 0.07)$ & $0.21( \pm 0.003)$ & 0.73 & 0.19 & 0.21 & $0.73( \pm 0.07)$ & 0.89 & 0.37 & 0.75 & $1.32( \pm 0.12)$ & 1.59 & 0.73 & 1.35 \\
\hline $\begin{array}{l}\mathrm{G} 168.72- \\
15.48-\mathrm{H} 2\end{array}$ & $6.8( \pm 0.6)$ & 7.8 & 5.9 & 6.9 & $14.9( \pm 0.7)$ & 16.6 & 13.7 & 14.9 & $0.84( \pm 0.07)$ & $0.21( \pm 0.005)$ & 0.22 & 0.20 & 0.21 & $0.67( \pm 0.09)$ & 0.82 & 0.43 & 0.67 & $1.21( \pm 0.15)$ & 1.47 & 0.84 & 1.21 \\
\hline $\begin{array}{l}\text { G169.43- } \\
16.17-\mathrm{H} 1\end{array}$ & $6.1( \pm 0.4)$ & 7.0 & 5.3 & 6.0 & $11.0( \pm 0.4)$ & 12.1 & 10.1 & 11.0 & $1.07( \pm 0.10)$ & $0.18( \pm 0.003)$ & 0.19 & 0.18 & 0.18 & $0.58( \pm 0.05)$ & 0.66 & 0.46 & 0.59 & $1.05( \pm 0.08)$ & 1.19 & 0.85 & 1.07 \\
\hline $\begin{array}{l}\text { G169.43- } \\
16.17-\mathrm{H} 2\end{array}$ & $1.8( \pm 0.3)$ & 2.1 & 1.1 & 1.8 & $11.9( \pm 0.8)$ & 13.7 & 10.6 & 11.8 & $1.07( \pm 0.17)$ & $0.19( \pm 0.006)$ & 0.19 & 0.18 & 0.19 & $0.40( \pm 0.16)$ & 0.70 & 0.46 & 0.65 & $0.77( \pm 0.24)$ & 1.27 & 1.00 & 1.18 \\
\hline $\begin{array}{l}\text { G169.76- } \\
16.15-\mathrm{H} 1\end{array}$ & $5.3( \pm 0.4)$ & 5.9 & 4.5 & 5.2 & $11.2( \pm 0.6)$ & 12.7 & 10.1 & 11.0 & $1.09( \pm 0.15)$ & $0.18( \pm 0.005)$ & 0.20 & 0.18 & 0.18 & $0.53( \pm 0.04)$ & 0.60 & 0.47 & 0.53 & $0.98( \pm 0.07)$ & 1.09 & 0.87 & 0.97 \\
\hline $\begin{array}{l}\text { G169.76- } \\
16.15-\mathrm{H} 2\end{array}$ & $4.8( \pm 0.2)$ & 5.2 & 4.4 & 4.9 & $11.0( \pm 0.6)$ & 12.4 & 10.1 & 10.8 & $0.85( \pm 0.10)$ & $0.18( \pm 0.005)$ & 0.19 & 0.18 & 0.18 & $0.47( \pm 0.04)$ & 0.56 & 0.41 & 0.47 & $0.88( \pm 0.06)$ & 1.01 & 0.78 & 0.86 \\
\hline $\begin{array}{c}\text { G169.76- } \\
16.15-\mathrm{H} 3\end{array}$ & $3.5( \pm 0.3)$ & 4.1 & 2.9 & 3.5 & $12.9( \pm 0.9)$ & 14.4 & 10.6 & 13.0 & $0.48( \pm 0.06)$ & $0.20( \pm 0.007)$ & 0.19 & 0.18 & 0.18 & $0.51( \pm 0.07)$ & 0.54 & 0.38 & 0.47 & $0.95( \pm 0.11)$ & 0.99 & 0.74 & 0.87 \\
\hline $\begin{array}{l}\text { G169.76- } \\
16.15-\mathrm{H} 4\end{array}$ & $3.2( \pm 0.2)$ & 3.8 & 2.8 & 3.2 & $11.6( \pm 0.7)$ & 12.5 & 10.2 & 11.8 & $0.66( \pm 0.08)$ & $0.19( \pm 0.006)$ & 0.20 & 0.18 & 0.19 & $0.49( \pm 0.11)$ & 0.59 & 0.27 & 0.48 & $0.91( \pm 0.18)$ & 1.06 & 0.57 & 0.89 \\
\hline $\begin{array}{l}\text { G169.76- } \\
\text { 16.15-H5 }\end{array}$ & $2.5( \pm 0.3)$ & 3.0 & 2.0 & 2.6 & $10.2( \pm 0.7)$ & 11.2 & 9.4 & 10.3 & $0.81( \pm 0.13)$ & $0.18( \pm 0.005)$ & 0.20 & 0.18 & 0.19 & $0.45( \pm 0.14)$ & 0.57 & 0.37 & 0.45 & $0.84( \pm 0.23)$ & 1.05 & 0.72 & 0.85 \\
\hline $\begin{array}{l}\text { G170.00- } \\
16.14-\mathrm{H} 1\end{array}$ & $3.7( \pm 0.3)$ & 4.0 & 3.2 & 3.8 & $11.4( \pm 0.6)$ & 12.4 & 10.4 & 11.7 & $0.59( \pm 0.06)$ & $0.19( \pm 0.005)$ & 0.20 & 0.18 & 0.19 & $0.52( \pm 0.18)$ & 0.69 & 0.16 & 0.64 & $0.97( \pm 0.28)$ & 1.24 & 0.42 & 1.16 \\
\hline $\begin{array}{l}\text { G170.00- } \\
16.14-\mathrm{H} 2\end{array}$ & $3.8( \pm 0.7)$ & 4.7 & 2.4 & 3.7 & $11.6( \pm 0.8)$ & 13.8 & 10.7 & 11.4 & $0.76( \pm 0.11)$ & $0.19( \pm 0.007)$ & 0.21 & 0.18 & 0.19 & $0.59( \pm 0.10)$ & 0.67 & 0.31 & 0.62 & $1.07( \pm 0.16)$ & 1.20 & 0.62 & 1.12 \\
\hline $\begin{array}{l}\text { G170.13- } \\
16.06-\mathrm{H} 1\end{array}$ & $5.0( \pm 0.4)$ & 5.9 & 3.9 & 5.0 & $11.4( \pm 0.7)$ & 12.8 & 10.1 & 11.5 & $0.72( \pm 0.09)$ & $0.19( \pm 0.006)$ & 0.20 & 0.18 & 0.19 & $0.55( \pm 0.04)$ & 0.63 & 0.44 & 0.56 & $1.01( \pm 0.07)$ & 1.13 & 0.82 & 1.02 \\
\hline $\begin{array}{l}\text { G170.26- } \\
16.02-\mathrm{H} 1\end{array}$ & $3.9( \pm 0.3)$ & 4.2 & 3.6 & 3.8 & $11.4( \pm 0.6)$ & 12.3 & 10.6 & 11.4 & $1.01( \pm 0.12)$ & $0.18( \pm 0.005)$ & 0.22 & 0.16 & 0.18 & $0.43( \pm 0.02)$ & 0.77 & 0.30 & 0.43 & $0.82( \pm 0.08)$ & 1.38 & 0.60 & 0.82 \\
\hline $\begin{array}{l}\text { G170.26- } \\
16.02-\mathrm{H} 2\end{array}$ & $4.2( \pm 0.1)$ & 4.5 & 3.9 & 4.2 & $10.6( \pm 0.5)$ & 11.4 & 9.7 & 10.7 & $1.42( \pm 0.21)$ & $0.18( \pm 0.004)$ & 0.19 & 0.17 & 0.18 & $0.46( \pm 0.05)$ & 0.54 & 0.32 & 0.48 & $0.86( \pm 0.09)$ & 0.98 & 0.63 & 0.88 \\
\hline $\begin{array}{l}\text { G170.83- } \\
15.90-\mathrm{H} 1\end{array}$ & $1.7( \pm 0.4)$ & 2.4 & 9.3 & 1.7 & $9.6( \pm 0.8)$ & 11.4 & 8.5 & 9.5 & $1.81( \pm 0.65)$ & $0.17( \pm 0.007)$ & 0.19 & 0.16 & 0.17 & $0.39( \pm 0.14)$ & 0.58 & 0.07 & 0.41 & $0.74( \pm 0.22)$ & 1.05 & 0.31 & 0.76 \\
\hline $\begin{array}{l}\text { G170.83- } \\
15.90-\mathrm{H} 2\end{array}$ & $1.3( \pm 0.4)$ & 2.3 & 3.2 & 1.3 & $10.2( \pm 0.9)$ & 12.4 & 8.1 & 10.2 & $1.28( \pm 0.32)$ & $0.18( \pm 0.008)$ & 0.19 & 0.16 & 0.18 & $0.37( \pm 0.11)$ & 0.69 & 0.11 & 0.39 & $0.72( \pm 0.18)$ & 1.24 & 0.34 & 0.73 \\
\hline $\begin{array}{l}\text { G170.99- } \\
15.81-\mathrm{H} 1\end{array}$ & $3.8( \pm 0.9)$ & 5.8 & 2.0 & 3.7 & $8.9( \pm 1.0)$ & 11.6 & 7.1 & 8.8 & $1.89( \pm 1.01)$ & $0.16( \pm 0.010)$ & 0.19 & 0.15 & 0.16 & $0.58( \pm 0.18)$ & 0.94 & 0.14 & 0.61 & $1.05( \pm 0.30)$ & 1.65 & 0.38 & 1.09 \\
\hline $\begin{array}{l}\text { G171.49- } \\
\text { 14.90-H1 }\end{array}$ & $4.5( \pm 0.5)$ & 5.1 & 3.8 & 4.5 & $12.4( \pm 0.8)$ & 14.0 & 11.6 & 12.2 & $0.88( \pm 0.12)$ & $0.19( \pm 0.006)$ & 0.20 & 0.18 & 0.18 & $0.51( \pm 0.08)$ & 0.54 & 0.14 & 0.38 & $0.95( \pm 0.13)$ & 0.99 & 0.39 & 0.73 \\
\hline $\begin{array}{l}\text { G171.80- } \\
\quad 15.32-\mathrm{H} 1\end{array}$ & $3.9( \pm 0.5)$ & 4.5 & 3.3 & 3.8 & $12.1( \pm 0.7)$ & 13.6 & 11.0 & 12.0 & $1.05( \pm 0.14)$ & $0.19( \pm 0.005)$ & 0.20 & 0.18 & 0.19 & $0.43( \pm 0.13)$ & 0.66 & 0.20 & 0.44 & $0.81( \pm 0.20)$ & 1.18 & 0.48 & 0.83 \\
\hline $\begin{array}{l}\text { G171.80- } \\
15.32-\mathrm{H} 2\end{array}$ & $4.5( \pm 0.3)$ & 5.0 & 4.2 & 4.5 & $11.4( \pm 0.6)$ & 12.9 & 10.6 & 11.5 & $1.02( \pm 0.13)$ & $0.19( \pm 0.005)$ & 0.20 & 0.18 & 0.19 & $0.65( \pm 0.06)$ & 0.75 & 0.46 & 0.66 & $1.16( \pm 0.11)$ & 1.32 & 0.86 & 1.19 \\
\hline $\begin{array}{l}\text { G171.80- } \\
15.32-\mathrm{H} 3\end{array}$ & $3.7( \pm 0.3)$ & 4.2 & 3.1 & 3.6 & $10.8( \pm 0.4)$ & 11.6 & 10.3 & 10.7 & $1.14( \pm 0.10)$ & $0.18( \pm 0.003)$ & 0.19 & 0.18 & 0.18 & $0.66( \pm 0.07)$ & 0.76 & 0.53 & 0.66 & $1.19( \pm 0.11)$ & 1.36 & 0.97 & 1.18 \\
\hline $\begin{array}{l}\text { G171.91- } \\
15.65-\mathrm{H} 1\end{array}$ & $4.8( \pm 0.3)$ & 5.3 & 4.3 & 4.6 & $11.4( \pm 0.5)$ & 12.3 & 10.8 & 11.4 & $1.49( \pm 0.19)$ & $0.19( \pm 0.004)$ & 0.19 & 0.18 & 0.19 & $0.42( \pm 0.09)$ & 0.52 & 0.27 & 0.43 & $0.80( \pm 0.13)$ & 0.96 & 0.57 & 0.81 \\
\hline
\end{tabular}


Table 6

(Continued)

\begin{tabular}{|c|c|c|c|c|c|c|c|c|c|c|c|c|c|c|c|c|c|c|c|c|c|}
\hline \multirow{2}{*}{ Name } & \multicolumn{4}{|c|}{$N_{\mathrm{H}_{2}}(\mathrm{PMO})$} & \multicolumn{4}{|c|}{$T_{\text {ex }}$} & \multirow{2}{*}{$\tau\left({ }^{13} \mathrm{CO}\right)$} & \multicolumn{4}{|c|}{$\sigma_{\text {th }}$} & \multicolumn{4}{|c|}{$\sigma_{\mathrm{NT}}\left({ }^{13} \mathrm{CO}\right)$} & \multicolumn{4}{|c|}{$\sigma_{3 \mathrm{D}}\left({ }^{13} \mathrm{CO}\right)$} \\
\hline & mean & $\begin{array}{r}\max \\
\left(10^{21}\right.\end{array}$ & $\left.\min _{-2}\right)$ & median & mean & $\underset{(\mathrm{max})}{(\mathrm{n})}$ & $\min$ & median & & mean & $\max _{\left(\mathrm{km} \mathrm{s}^{-1}\right)}$ & $\min$ & median & mean & $\begin{array}{l}\max _{\left(\mathrm{km} \mathrm{s}^{-1}\right)} \\
\text {. }\end{array}$ & $\min$ & median & mean & $\begin{array}{l}\max _{\left(\mathrm{km} \mathrm{s}^{-1}\right)} \\
\text {. }\end{array}$ & $\min$ & median \\
\hline $\begin{array}{l}\text { G171.91- } \\
15.65-\mathrm{H} 2\end{array}$ & $4.6( \pm 0.5)$ & 5.3 & 3.6 & 4.7 & $11.3( \pm 0.4)$ & 12.1 & 10.9 & 11.3 & $1.64( \pm 0.19)$ & $0.19( \pm 0.003)$ & 0.19 & 0.18 & 0.19 & $0.43( \pm 0.08)$ & 0.53 & 0.27 & 0.43 & $0.80( \pm 0.14)$ & 0.97 & 0.13 & 0.80 \\
\hline $\begin{array}{l}\text { G171.91- } \\
15.65-\mathrm{H} 3\end{array}$ & $4.4( \pm 0.2)$ & 4.5 & 3.8 & 4.2 & $12.2( \pm 0.5)$ & 13.0 & 11.0 & 12.1 & $1.16( \pm 0.12)$ & $0.19( \pm 0.004)$ & 0.20 & 0.18 & 0.19 & $0.36( \pm 0.09)$ & 0.48 & 0.23 & 0.40 & $0.71( \pm 0.14)$ & 0.89 & 0.52 & 0.77 \\
\hline $\begin{array}{l}\text { G172.06- } \\
\quad 15.21-\mathrm{H} 1\end{array}$ & $3.7( \pm 0.3)$ & 4.3 & 3.2 & 3.7 & $13.3( \pm 0.4)$ & 13.9 & 12.4 & 13.2 & $0.71( \pm 0.05)$ & $0.20( \pm 0.003)$ & 0.21 & 0.20 & 0.20 & $0.41( \pm 0.06)$ & 0.52 & 0.31 & 0.41 & $0.79( \pm 0.09)$ & 0.97 & 0.64 & 0.79 \\
\hline
\end{tabular}

Note. All of these parameters are derived from the PMO observational data. Excitation temperature is calculated by ${ }^{12} \mathrm{CO}$ data, and $N_{\mathrm{H}_{2}}(\mathrm{PMO}), \sigma_{\mathrm{NT}}$, and $\sigma_{3 \mathrm{D}}$ are derived from ${ }^{13} \mathrm{CO}$ images. 
Table 7

The Parameters of $\mathrm{C}^{18} \mathrm{O}$ Molecules

\begin{tabular}{|c|c|c|c|c|c|c|}
\hline Name & $\begin{array}{l}N_{\mathrm{C}^{18} \mathrm{O}}(\mathrm{LTE}) \\
\left(10^{14} \mathrm{~cm}^{-2}\right)\end{array}$ & $\begin{array}{c}\mathrm{C}^{18} \mathrm{O} \text { Abundance(LTE) } \\
\left(10^{-7}\right)\end{array}$ & $f_{\mathrm{D}}$ & $\tau\left(\mathrm{C}^{18} \mathrm{O}\right)$ & $\begin{array}{c}T_{\mathrm{ex}}(\mathrm{non}-\mathrm{LTE})\left(\mathrm{C}^{18} \mathrm{O}\right) \\
(\mathrm{K})\end{array}$ & $\begin{array}{l}N_{\mathrm{C}^{18} \mathrm{O}}(\text { non-LTE }) \\
\quad\left(10^{15} \mathrm{~cm}^{-2}\right)\end{array}$ \\
\hline G166.99-15.34-H1 & $1.59( \pm 0.22)$ & $0.44( \pm 0.18)$ & $6.59( \pm 1.75)$ & 0.02 & 13.99 & 0.15 \\
\hline G167.23-15.32-H1 & $4.92( \pm 0.40)$ & $0.66( \pm 0.26)$ & $4.36( \pm 1.16)$ & 0.10 & 13.02 & 0.18 \\
\hline G168.13-16.39-H1 & $19.63( \pm 0.17)$ & $1.28( \pm 0.34)$ & $2.24( \pm 0.59)$ & 0.38 & 12.06 & 2.54 \\
\hline G168.13-16.39-H2 & $17.16( \pm 0.15)$ & $1.29( \pm 0.15)$ & $2.22( \pm 0.59)$ & 0.28 & 11.80 & 2.02 \\
\hline G168.72-15.48-H1 & $17.62( \pm 0.19)$ & $1.26( \pm 0.21)$ & $2.28( \pm 0.61)$ & 0.37 & 12.08 & 2.24 \\
\hline G169.43-16.17-H2 & $14.58( \pm 0.36)$ & $1.48( \pm 0.31)$ & $1.95( \pm 0.52)$ & 0.20 & 12.12 & 1.66 \\
\hline G169.76-16.15-H1 & $22.89( \pm 0.23)$ & $2.35( \pm 0.31)$ & $1.22( \pm 0.32)$ & 0.24 & 12.19 & 2.69 \\
\hline G169.76-16.15-H2 & $22.05( \pm 0.10)$ & $2.31( \pm 0.23)$ & $1.24( \pm 0.33)$ & 0.24 & 12.53 & 2.54 \\
\hline G169.76-16.15-H3 & $8.42( \pm 0.15)$ & $0.80( \pm 0.21)$ & $3.58( \pm 0.95)$ & 0.14 & 12.43 & 0.96 \\
\hline G169.76-16.15-H4 & $9.23( \pm 0.15)$ & $0.83( \pm 0.19)$ & $3.46( \pm 0.92)$ & 0.21 & 12.05 & 1.10 \\
\hline G169.76-16.15-H5 & $6.25( \pm 0.16)$ & $0.55( \pm 0.18)$ & $5.20( \pm 1.38)$ & 0.16 & 12.20 & 0.74 \\
\hline G170.26-16.02-H2 & $1.22( \pm 0.22)$ & $0.99( \pm 0.32)$ & $2.91( \pm 0.77)$ & 0.22 & 11.23 & 1.52 \\
\hline G170.83-15.90-H1 & $15.08( \pm 0.41)$ & $2.87( \pm 0.76)$ & $1.00( \pm 0.27)$ & 0.16 & 12.55 & 1.59 \\
\hline G170.83-15.90-H2 & $10.04( \pm 0.49)$ & $1.86( \pm 0.36)$ & $1.54( \pm 0.41)$ & 0.13 & 12.66 & 1.09 \\
\hline G170.99-15.81-H1 & $13.12( \pm 0.28)$ & $1.61( \pm 0.40)$ & $1.79( \pm 0.47)$ & 0.30 & 11.72 & 1.48 \\
\hline G171.49-14.90-H1 & $10.21( \pm 0.34)$ & $0.48( \pm 0.17)$ & $5.97( \pm 1.58)$ & 0.36 & 11.35 & 1.44 \\
\hline G171.80-15.32-H1 & $9.82( \pm 0.41)$ & $0.66( \pm 0.25)$ & $4.37( \pm 1.16)$ & 0.12 & 11.24 & 1.17 \\
\hline G171.80-15.32-H2 & $10.82( \pm 1.44)$ & $1.21( \pm 0.37)$ & $2.36( \pm 0.63)$ & 0.08 & 12.76 & 1.23 \\
\hline G171.80-15.32-H3 & $11.82( \pm 0.29)$ & $0.88( \pm 0.11)$ & $3.25( \pm 0.86)$ & 0.06 & 11.37 & 0.72 \\
\hline G171.91-15.65-H1 & $12.82( \pm 0.51)$ & $1.64( \pm 0.60)$ & $1.75( \pm 0.46)$ & 0.14 & 14.10 & 1.48 \\
\hline G171.91-15.65-H2 & $13.82( \pm 0.66)$ & $1.42( \pm 0.68)$ & $2.03( \pm 0.54)$ & 0.21 & 13.47 & 1.80 \\
\hline G171.91-15.65-H3 & $14.82( \pm 0.13)$ & $1.80( \pm 0.17)$ & $1.60( \pm 0.42)$ & 0.20 & 12.69 & 1.26 \\
\hline G172.06-15.21-H1 & $15.82( \pm 0.17)$ & $1.83( \pm 0.35)$ & $1.57( \pm 0.42)$ & 0.22 & 12.39 & 1.13 \\
\hline
\end{tabular}

Note. The LTE parameters are derived from $\mathrm{C}^{18} \mathrm{O}$ spectra. The non-LTE parameters and $\tau\left(\mathrm{C}^{18} \mathrm{O}\right)$ are derived from the "Radex" software. The depletion factor $\left(f_{D}\right)$ is calculated by $N_{\mathrm{C}^{18} \mathrm{O}}(\mathrm{LTE})$.

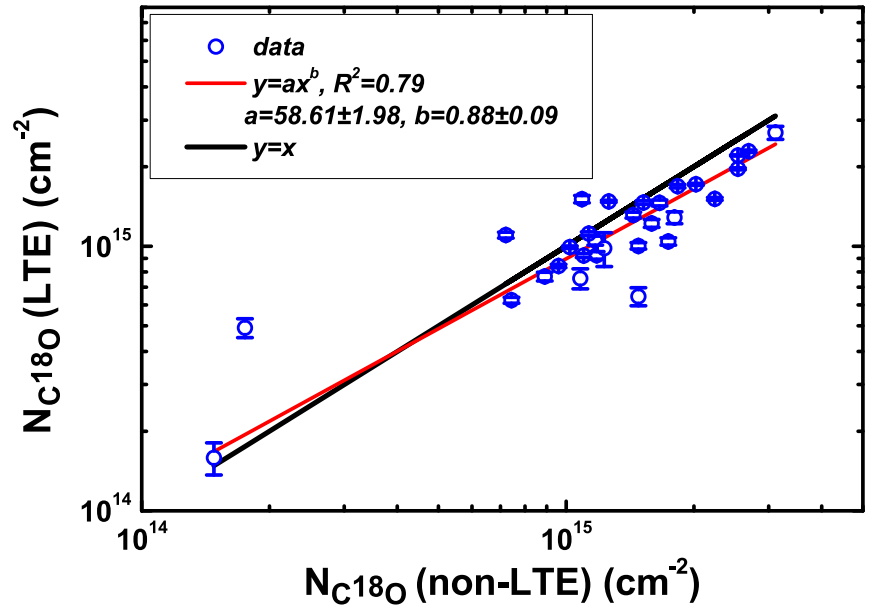

Figure 6. Relationship of the LTE $\mathrm{C}^{18} \mathrm{O}$ column density and the non-LTE $\mathrm{C}^{18} \mathrm{O}$ column density for all of the 30 dense cores in PGCCs. The red solid line is a power-law fit. Each blue dot represents a single dense core. The black solid line is $N_{\mathrm{C}^{18} \mathrm{O}}(\mathrm{LTE})=N_{\mathrm{C}^{18} \mathrm{O}}$ (non-LTE). The models are presented in the upper left corner of the figure. A strong correlation can be seen very close to $N_{\mathrm{C}^{18} \mathrm{O}}$ $(\mathrm{LTE})=N_{\mathrm{C}^{18} \mathrm{O}}$ (non-LTE), which indicates that the LTE $\mathrm{C}^{18} \mathrm{O}$ column density estimates are reasonable.

than 2 and hence are gravitationally bound and may collapse. In Figure 7, we plot $M_{\text {core }}$ against $M_{\text {vir }}$ and the black dashed line indicates $M_{\mathrm{vir}}=2 M_{\text {core }}$. We find that some protostellar cores and prestellar core candidates have virial parameters larger than 2. This apparent discrepancy might be due to the following factors:

1. Turbulence contributions. Turbulence is only dissipated in the densest regions of clouds, while the PMO observations cannot resolve such small-scale dense regions. The size of the $\mathrm{C}^{18} \mathrm{O}$ core can be larger than the Herschel continuum core such that the $\mathrm{C}^{18} \mathrm{O}$ line width may include significant contributions from its turbulent surroundings. For example, Pattle et al. (2015) found that non-thermal line widths decrease substantially between the gas traced by $\mathrm{C}^{18} \mathrm{O}$ and that traced by $\mathrm{N}_{2} \mathrm{H}^{+}$ in the dense cores of the Ophiuchus molecular cloud, indicating the dissipation of turbulence at higher densities.

2. $\mathrm{C}^{18} \mathrm{O}$ depletion. The depletion of $\mathrm{C}^{18} \mathrm{O}$ at the inner core region makes the $\mathrm{C}^{18} \mathrm{O}(1-0)$ line a poor tracer at those locations. Thus, the $\mathrm{C}^{18} \mathrm{O}$ line width is determined more by the gaseous outer, more turbulent regions. The presence of a central region affected by strong $\mathrm{CO}$ depletion could be the most important factor of uncertainty in the virial parameter (Giannetti et al. 2014).

Both of these factors can lead to an overestimation of the virial parameter. $\mathrm{C}^{18} \mathrm{O}$ lines, however, have often been used for virial analysis. For comparison, we plot the data from Onishi et al. (1996), Tachihara et al. (2000), and Zhang et al. (2015) in gray stars, orange circles, and black triangles, 
Table 8

The Parameters of the Dense Cores

\begin{tabular}{|c|c|c|c|c|c|c|}
\hline Name & $\begin{array}{c}V_{\mathrm{lsr}}\left(\mathrm{C}^{18} \mathrm{O}\right) \\
\left(\mathrm{km} \mathrm{s}^{-1}\right)\end{array}$ & $\begin{array}{l}M_{\mathrm{vir}} \\
\left(M_{\odot}\right)\end{array}$ & $\alpha$ & $\begin{array}{c}\text { Jeans Length } \\
(\mathrm{pc})\end{array}$ & $\begin{array}{c}\sigma_{\mathrm{NT}}\left(\mathrm{C}^{18} \mathrm{O}\right) \\
\left(\mathrm{km} \mathrm{s}^{-1}\right)\end{array}$ & Mach Number \\
\hline G166.99-15.34-H1 & $6.93( \pm 0.10)$ & $23.49( \pm 17.76)$ & $6.50( \pm 5.20)$ & $0.375( \pm 0.011)$ & $0.43( \pm 0.32)$ & $2.66( \pm 2.05)$ \\
\hline G167.23-15.32-H1 & $6.65( \pm 0.04)$ & $6.48( \pm 3.06)$ & $0.97( \pm 0.49)$ & $0.249( \pm 0.007)$ & $0.22( \pm 0.03)$ & $1.22( \pm 0.20)$ \\
\hline G168.00-15.69-H1 & $7.79( \pm 0.01)$ & $16.82( \pm 1.21)$ & $0.58( \pm 0.04)$ & $0.440( \pm 0.010)$ & $0.23( \pm 0.01)$ & $1.04( \pm 0.05)$ \\
\hline G168.13-16.39-H1 & $6.60( \pm 0.01)$ & $20.20( \pm 1.02)$ & $0.58( \pm 0.03)$ & $0.207( \pm 0.006)$ & $0.32( \pm 0.01)$ & $1.65( \pm 0.07)$ \\
\hline G168.13-16.39-H2 & $6.84( \pm 0.02)$ & $35.58( \pm 3.31)$ & $0.98( \pm 0.09)$ & $0.244( \pm 0.004)$ & $0.37( \pm 0.02)$ & $1.92( \pm 0.14)$ \\
\hline G168.72-15.48-H1 & $7.37( \pm 0.02)$ & $16.66( \pm 1.22)$ & $0.63( \pm 0.05)$ & $0.214( \pm 0.005)$ & $0.28( \pm 0.01)$ & $1.37( \pm 0.05)$ \\
\hline G168.72-15.48-H2 & $7.31( \pm 0.01)$ & $9.49( \pm 1.27)$ & $0.25( \pm 0.03)$ & $0.164( \pm 0.013)$ & $0.24( \pm 0.01)$ & $1.11( \pm 0.06)$ \\
\hline G169.43-16.17-H1 & $5.53( \pm 0.03)$ & $74.28( \pm 7.64)$ & $2.45( \pm 0.26)$ & $0.275( \pm 0.006)$ & $0.56( \pm 0.15)$ & $3.04( \pm 0.89)$ \\
\hline G169.43-16.17-H2 & $5.64( \pm 0.03)$ & $28.21( \pm 5.30)$ & $2.28( \pm 0.45)$ & $0.231( \pm 0.005)$ & $0.42( \pm 0.05)$ & $2.19( \pm 0.31)$ \\
\hline G169.76-16.15-H1 & $5.69( \pm 0.02)$ & $37.45( \pm 3.46)$ & $5.78( \pm 0.62)$ & $0.205( \pm 0.004)$ & $0.55( \pm 0.19)$ & $2.96( \pm 1.10)$ \\
\hline G169.76-16.15-H2 & $5.83( \pm 0.02)$ & $32.81( \pm 2.56)$ & $4.64( \pm 0.42)$ & $0.222( \pm 0.002)$ & $0.49( \pm 0.04)$ & $2.69( \pm 0.30)$ \\
\hline G169.76-16.15-H3 & $6.90( \pm 0.01)$ & $11.85( \pm 1.15)$ & $1.26( \pm 0.14)$ & $0.202( \pm 0.005)$ & $0.30( \pm 0.01)$ & $1.53( \pm 0.11)$ \\
\hline G169.76-16.15-H4 & $6.88( \pm 0.01)$ & $7.97( \pm 0.78)$ & $0.91( \pm 0.10)$ & $0.189( \pm 0.005)$ & $0.25( \pm 0.01)$ & $1.33( \pm 0.09)$ \\
\hline G169.76-16.15-H5 & $6.90( \pm 0.02)$ & $3.86( \pm 0.73)$ & $0.87( \pm 0.20)$ & $0.157( \pm 0.004)$ & $0.21( \pm 0.01)$ & $1.19( \pm 0.10)$ \\
\hline G170.00-16.14-H1 & $6.59( \pm 0.08)$ & $16.00( \pm 3.62)$ & $4.97( \pm 1.37)$ & $0.128( \pm 0.004)$ & $0.47( \pm 0.09)$ & $2.54( \pm 0.56)$ \\
\hline G170.00-16.14-H2 & $6.61( \pm 0.05)$ & $16.68( \pm 3.01)$ & $3.66( \pm 0.81)$ & $0.143( \pm 0.009)$ & $0.47( \pm 0.07)$ & $2.51( \pm 0.46)$ \\
\hline G170.13-16.06-H1 & $6.85( \pm 0.03)$ & $25.16( \pm 4.34)$ & $0.94( \pm 0.17)$ & $0.240( \pm 0.007)$ & $0.34( \pm 0.03)$ & $1.82( \pm 0.19)$ \\
\hline G170.26-16.02-H1 & $6.64( \pm 0.04)$ & $20.28( \pm 2.53)$ & $15.08( \pm 3.43)$ & $0.146( \pm 0.004)$ & $0.60( \pm 0.10)$ & $3.28( \pm 0.63)$ \\
\hline G170.26-16.02-H2 & $6.56( \pm 0.01)$ & $12.67( \pm 1.07)$ & $1.63( \pm 0.17)$ & $0.153( \pm 0.006)$ & $0.35( \pm 0.02)$ & $1.97( \pm 0.13)$ \\
\hline G170.83-15.90-H1 & $6.57( \pm 0.04)$ & $35.56( \pm 7.05)$ & $7.09( \pm 1.66)$ & $0.305( \pm 0.007)$ & $0.50( \pm 0.23)$ & $2.92( \pm 1.48)$ \\
\hline G170.83-15.90-H2 & $6.79( \pm 0.07)$ & $18.27( \pm 6.41)$ & $4.53( \pm 1.68)$ & $0.267( \pm 0.006)$ & $0.40( \pm 0.09)$ & $2.30( \pm 0.61)$ \\
\hline G170.99-15.81-H1 & $6.51( \pm 0.02)$ & $26.87( \pm 3.84)$ & $0.45( \pm 0.07)$ & $0.377( \pm 0.009)$ & $0.27( \pm 0.02)$ & $1.66( \pm 0.20)$ \\
\hline G171.49-14.90-H1 & $6.55( \pm 0.02)$ & $3.60( \pm 0.65)$ & $0.36( \pm 0.07)$ & $0.111( \pm 0.004)$ & $0.19( \pm 0.01)$ & $1.00( \pm 0.08)$ \\
\hline G171.80-15.32-H1 & $6.96( \pm 0.06)$ & $25.72( \pm 5.42)$ & $3.27( \pm 0.77)$ & $0.134( \pm 0.005)$ & $0.52( \pm 0.12)$ & $2.72( \pm 0.72)$ \\
\hline G171.80-15.32-H2 & $7.38( \pm 0.13)$ & $35.66( \pm 12.67)$ & $17.46( \pm 7.27)$ & $0.167( \pm 0.010)$ & $0.71( \pm 0.21)$ & $3.83( \pm 1.20)$ \\
\hline G171.80-15.32-H3 & 7.12( \pm 0.11$)$ & $50.51( \pm 19.20)$ & $4.47( \pm 1.75)$ & $0.169( \pm 0.004)$ & $0.62( \pm 0.25)$ & $3.40( \pm 1.42)$ \\
\hline G171.91-15.65-H1 & $6.56( \pm 0.04)$ & $7.50( \pm 1.45)$ & $6.17( \pm 2.15)$ & $0.169( \pm 0.005)$ & $0.38( \pm 0.04)$ & $2.06( \pm 0.24)$ \\
\hline G171.91-15.65-H2 & $6.64( \pm 0.03)$ & $6.40( \pm 0.96)$ & $2.56( \pm 0.58)$ & $0.157( \pm 0.007)$ & $0.33( \pm 0.02)$ & $1.76( \pm 0.13)$ \\
\hline G171.91-15.65-H3 & $6.66( \pm 0.02)$ & $9.61( \pm 1.53)$ & $2.27( \pm 0.41)$ & $0.218( \pm 0.002)$ & $0.29( \pm 0.02)$ & $1.50( \pm 0.11)$ \\
\hline G172.06-15.21-H1 & $6.81( \pm 0.01)$ & $7.30( \pm 0.79)$ & $0.94( \pm 0.12)$ & $0.219( \pm 0.006)$ & $0.24( \pm 0.01)$ & $1.18( \pm 0.05)$ \\
\hline
\end{tabular}

Note. $\alpha$ is calculated by $M_{\text {vir }} / M_{\text {core }}$, and the other parameters are derived from $\mathrm{C}^{18} \mathrm{O}$ data.

${ }^{\mathrm{a}}$ The Mach numbers are derived from $\sigma_{\mathrm{NT}}\left(\mathrm{C}^{18} \mathrm{O}\right) / \sigma_{\mathrm{th}}$.

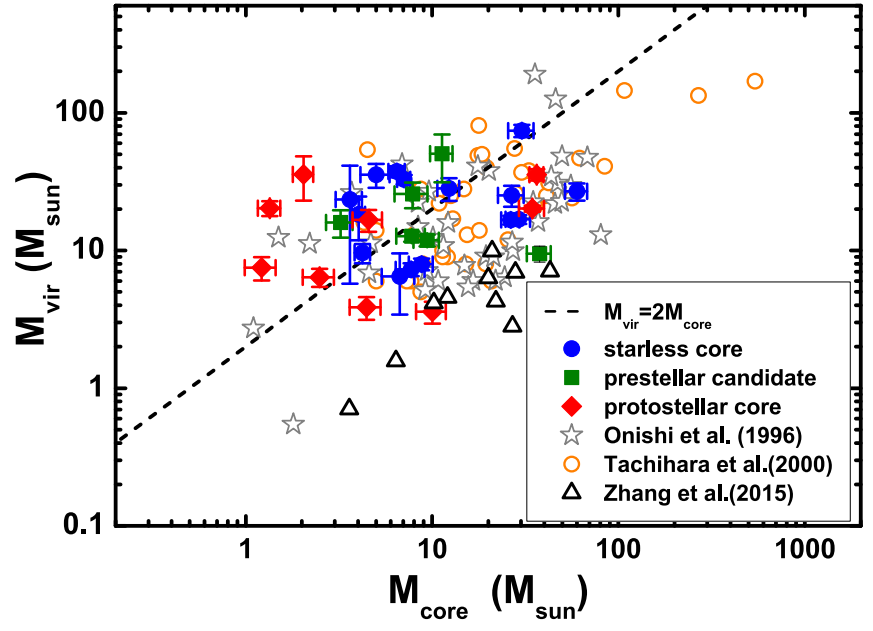

Figure 7. Relationship between the $M_{\text {core }}$ and $M_{\text {vir }}$ of dense cores. The red diamonds, green squares, and blue dots represent the protostellar cores, prestellar core candidates, and starless cores, respectively. The gray stars, orange circles, and black triangles are data from Onishi et al. (1996), Tachihara et al. (2000), and Zhang et al. (2015), respectively. The black dashed line indicates $M_{\mathrm{vir}}=2 M_{\text {core. }}$. The virial parameter $\alpha=2$ can be regarded as a lower limit for gas motions to prevent collapse.

respectively, in Figures 7 and 8. The virial masses in those works were also calculated with $\mathrm{C}^{18} \mathrm{O}$ lines. In contrast to dense cores in other works, the cores in L1495 have similar but relatively larger virial parameters. We found that dense cores in far-away infrared dark clouds (Zhang et al. 2015; Ohashi et al. 2016; Sanhueza et al. 2017) have much smaller virial parameters than Taurus cores, suggesting that infrared dark clouds have much denser environments for star formation. A survey by Fehér et al. (2017) found that 5 out of 21 PGCCs are gravitationally bound, based on Hersche$l$ and $\mathrm{CO}$ data.

As we mentioned before, the turbulence is dissipated in denser regions, $\mathrm{C}^{18} \mathrm{O}$ is affected by turbulence from outer regions, and turbulence is also significantly affected by $\mathrm{C}^{18} \mathrm{O}$ depletion. Observations of this region in nitrogen-bearing tracers with comparable resolution were presented by Seo et al. (2015). A total of 12 dense cores in our samples correspond to theirs. The mean value of non-thermal velocity dispersion derived from their $\mathrm{NH}_{3}$ and our $\mathrm{C}^{18} \mathrm{O}$ are $0.20( \pm 0.02) \mathrm{km} \mathrm{s}^{-1}$ and $0.39 \pm$ $0.15 \mathrm{~km} \mathrm{~s}^{-1}$, respectively. This suggests that turbulence is considerably dissipated in more centered regions. The $\mathrm{C}^{18} \mathrm{O}$ in these regions was depleted, and non-thermal velocity dispersion derived from $\mathrm{C}^{18} \mathrm{O}$ mainly came from from diffused gas. Together with the above comparison, $\mathrm{C}^{18} \mathrm{O}$ lines may be not good indicators of the virial parameter, especially for prestellar and protostellar cores. To clarify the situation, high-resolution observations of dense gas tracers (e.g., $\mathrm{N}_{2} \mathrm{H}^{+}$) are needed.

Kauffmann et al. (2013) compiled a catalog containing 1325 virial estimates for entire molecular clouds ( $\gg 1 \mathrm{pc}$ scale), 


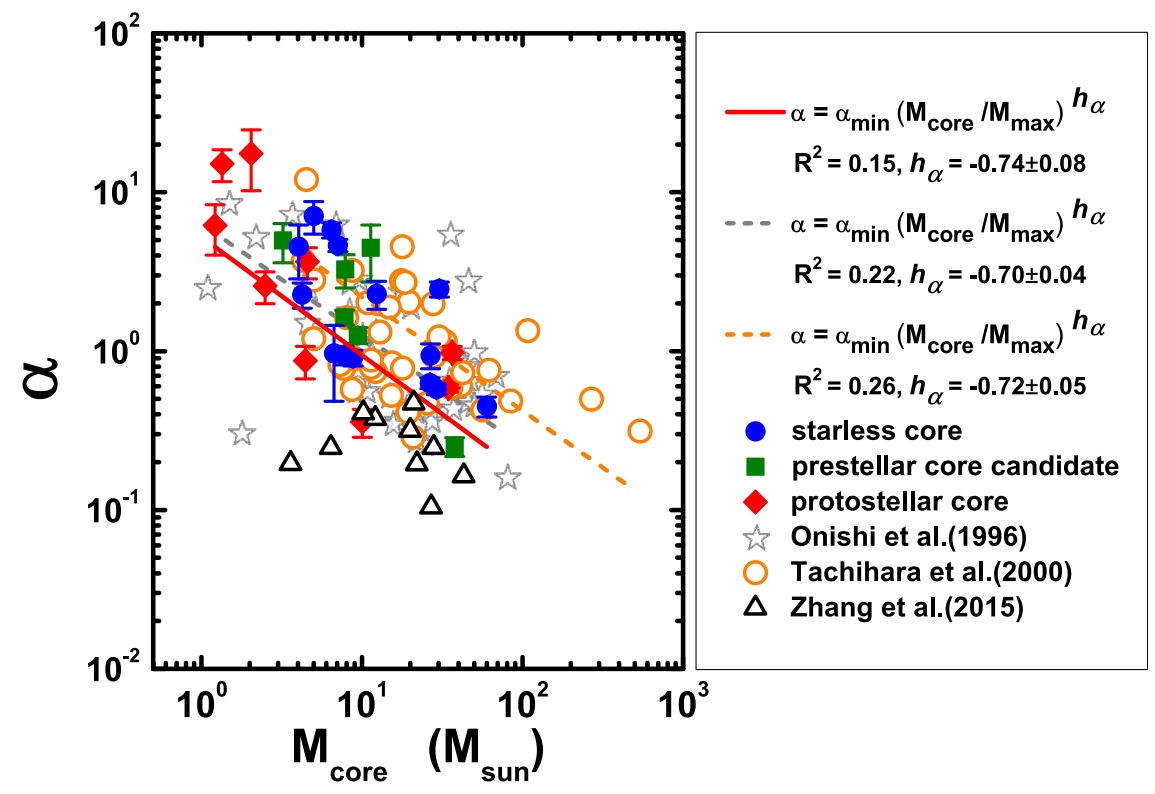

Figure 8. Relationship between $M_{\text {core }}$ and $\alpha$. The red diamonds, green squares, and blue dots represent the protostellar cores, prestellar core candidates, and starless cores, respectively. The gray stars, orange circles, and black triangles are data from Onishi et al. (1996), Tachihara et al. (2000), and Zhang et al. (2015) respectively. The red solid, gray, and orange dashed lines indicate power-law fitting of $\alpha=\alpha_{\min } \cdot\left(M_{\text {core }} / M_{\max }\right)^{h_{\alpha}}$ for L1495 samples, gray stars, and orange circles, respectively.

clumps $(\sim 1 \mathrm{pc})$, and cores $(\ll 1 \mathrm{pc})$. They suggested an anticorrelation exists between mass and virial parameter:

$$
\alpha=\alpha_{0} \cdot\left(M / 10^{3} M_{\odot}\right)^{h_{\alpha}},
$$

with a similar slope $h_{\alpha}$, and $\alpha_{0}$ is a range of intercepts. To highlight the trend, the equation above can be rewritten as

$$
\alpha=\alpha_{\min } \cdot\left(M / M_{\max }\right)^{h_{\alpha}},
$$

where $\alpha_{0}=\alpha_{\min } \cdot\left(M_{\max } / 10^{3} M_{\odot}\right)^{-h_{\alpha}}$. We fit this power-law function to our 30 samples and data from Onishi et al. (1996) and Tachihara et al. (2000); the fitting results are presented in Figure 8. Data from Zhang et al. (2015) were excluded from the fitting due to there being too few data points to fit. The virial parameters from our work, Onishi et al. (1996), and Tachihara et al. (2000) follow the above power-law behavior, with slopes $h_{\alpha}$ of $-0.74( \pm 0.08),-0.70( \pm 0.04)$, and $-0.72( \pm 0.05)$, respectively. This is consistent with the range $0<-h_{\alpha}<1$ reported in Kauffmann et al. (2013). Similar relationships between virial parameters and core masses were also reported by Loren (1989) and Bertoldi \& Mckee (1992). According to Figure 8 , we found that these cores also show similar powerlaw behaviors, indicating that these samples have similar physical properties. In Figures 7 and 8, the black triangles (Zhang et al. 2015) have the lowest virial parameters, and they indicate higher star-forming activity, such as outflows. This may indicate that our dense cores are at an evolutionary stage that is much earlier than those of the cores reported by Zhang et al. (2015).

We also calculated the thermal Jeans length (Jeans 1928) of dense cores; see Table 8 . We found that the radii of 30 dense cores are smaller than the thermal Jeans length. Considering that all dense core have supersonic non-thermal motions, this may indicate that the non-thermal motions are capable of creating an effectively hydrostatic pressure that opposes corecollapse (Mac Low \& Klessen 2004).

\subsection{Column Density Profile}

In this section, we study the density profiles of the dense cores. We averaged the Herschel column density data in concentric elliptical bins to get a column density profile, and then fitted the Spherical Geometry Model of Dapp \& Basu (2009). The underlying model resembles the Bonnor-Ebert model in that it features a flat central region, leading into a power-law decline $\propto r^{-2}$ in density, and a well-defined outer radius. The model can overcome the Bonnor-Ebert profile in several ways. However, this model does not assume that the cloud is in equilibrium, and can instead make qualitative statements about its dynamical state (expansion, equilibrium, collapse) using the size of the flat region as a proxy (Dapp \& Basu 2009). Therefore, it is more suitable to study the properties of dense cores in different dynamical states.

The basic model is

$$
n(r)= \begin{cases}n_{\mathrm{c}} a^{2} /\left(r^{2}+a^{2}\right) & r \leqslant R_{\mathrm{t}} \\ 0 & r>R_{\mathrm{t}}\end{cases}
$$

which is characterized by a central volume density $n_{\mathrm{c}}$ and a truncated radius $R_{\mathrm{t}}$ (To avoid confusion with the core radius from Gaussian fits, we use $R_{\mathrm{t}}$ to represent the truncated radius of cores derived from density profiles). The parameter $a$ represents the size of the inner flat region of a dense core. The column density $N_{x}$ can be derived by integrating the volume density along the line of sight through the sphere. Introducing the parameter $c \equiv R_{\mathrm{t}} / a, N_{\mathrm{c}}=2 a n_{\mathrm{c}} \arctan (c)$. Hence, the model can be rewritten as

$$
\begin{aligned}
N_{x}= & \frac{N_{\mathrm{c}}}{\sqrt{1+(x / a)^{2}}} \\
& \times\left[\arctan \left(\sqrt{\frac{c^{2}-(x / a)^{2}}{1+(x / a)^{2}}}\right) / \arctan (c)\right],
\end{aligned}
$$


Table 9

The Parameters Derived from Column Density Profiles

\begin{tabular}{|c|c|c|c|c|c|c|}
\hline Name & $\begin{array}{c}N_{\mathrm{c}} \\
\left(10^{22} \mathrm{~cm}^{-2}\right)\end{array}$ & $\begin{array}{l}R_{\mathrm{t}}{ }^{\mathrm{a}} \\
(\mathrm{pc})\end{array}$ & $\begin{array}{c}a \\
(\mathrm{pc})\end{array}$ & $\begin{array}{c}n_{\mathrm{c}}^{\mathrm{b}} \\
\left(10^{4} \mathrm{~cm}^{-3}\right)\end{array}$ & $\begin{array}{l}a_{\mathrm{d}}{ }^{\mathrm{c}} \\
(\mathrm{pc})\end{array}$ & $\begin{array}{l}R_{\mathrm{d}}{ }^{\mathrm{d}} \\
(\mathrm{pc})\end{array}$ \\
\hline G166.99-15.34-H1 & $0.66( \pm 0.01)$ & $0.12( \pm 0.04)$ & $0.028( \pm 0.002)$ & $2.88( \pm 0.38)$ & $0.025( \pm 0.002)$ & $0.12( \pm 0.04)$ \\
\hline G167.23-15.32-H1 & $1.19( \pm 0.01)$ & $0.10( \pm 0.01)$ & $0.045( \pm 0.001)$ & $3.77( \pm 0.12)$ & $0.043( \pm 0.001)$ & $0.10( \pm 0.01)$ \\
\hline G168.00-15.69-H1 & $0.91( \pm 0.01)$ & $0.28( \pm 0.04)$ & $0.089( \pm 0.005)$ & $1.31( \pm 0.11)$ & $0.089( \pm 0.005)$ & $0.28( \pm 0.04)$ \\
\hline G168.13-16.39-H1 & $2.68( \pm 0.01)$ & $0.38( \pm 0.04)$ & $0.038( \pm 0.002)$ & $7.79( \pm 0.45)$ & $0.036( \pm 0.002)$ & $0.40( \pm 0.04)$ \\
\hline G168.13-16.39-H2 & $1.62( \pm 0.01)$ & $0.25( \pm 0.03)$ & $0.043( \pm 0.006)$ & $4.35( \pm 0.64)$ & $0.042( \pm 0.006)$ & $0.25( \pm 0.03)$ \\
\hline G168.72-15.48-H1 & $2.10( \pm 0.01)$ & $0.93( \pm 0.67)$ & $0.048( \pm 0.017)$ & $4.65( \pm 1.75)$ & $0.047( \pm 0.017)$ & $0.92( \pm 0.67)$ \\
\hline G168.72-15.48-H2 & $4.51( \pm 0.02)$ & $0.37( \pm 0.25)$ & $0.028( \pm 0.001)$ & $17.74( \pm 1.30)$ & $0.025( \pm 0.001)$ & $0.37( \pm 0.25)$ \\
\hline G169.43-16.17-H1 & $1.51( \pm 0.01)$ & $0.19( \pm 0.04)$ & $0.083( \pm 0.007)$ & $2.56( \pm 0.35)$ & $0.082( \pm 0.007)$ & $0.19( \pm 0.04)$ \\
\hline G169.43-16.17-H2 & $1.41( \pm 0.01)$ & $0.09( \pm 0.04)$ & $0.039( \pm 0.017)$ & $5.02( \pm 2.23)$ & $0.037( \pm 0.018)$ & $0.09( \pm 0.04)$ \\
\hline G169.76-16.15-H1 & $1.20( \pm 0.01)$ & $0.88( \pm 0.44)$ & $0.048( \pm 0.006)$ & $2.65( \pm 0.30)$ & $0.047( \pm 0.005)$ & $0.88( \pm 0.44)$ \\
\hline G169.76-16.15-H2 & $1.13( \pm 0.01)$ & $0.99( \pm 0.36)$ & $0.053( \pm 0.005)$ & $2.26( \pm 0.24)$ & $0.052( \pm 0.005)$ & $0.99( \pm 0.36)$ \\
\hline G169.76-16.15-H3 & $1.80( \pm 0.01)$ & $0.29( \pm 0.11)$ & $0.029( \pm 0.001)$ & $6.95( \pm 0.43)$ & $0.026( \pm 0.001)$ & $0.29( \pm 0.11)$ \\
\hline G169.76-16.15-H4 & $1.73( \pm 0.01)$ & $0.29( \pm 0.23)$ & $0.029( \pm 0.002)$ & $6.50( \pm 0.72)$ & $0.027( \pm 0.002)$ & $0.29( \pm 0.23)$ \\
\hline G169.76-16.15-H5 & $2.18( \pm 0.03)$ & $0.10( \pm 0.05)$ & $0.018( \pm 0.001)$ & $14.06( \pm 1.93)$ & $0.014( \pm 0.002)$ & $0.10( \pm 0.05)$ \\
\hline G170.00-16.14-H1 & $1.86( \pm 0.02)$ & $0.09( \pm 0.02)$ & $0.026( \pm 0.002)$ & $9.11( \pm 1.03)$ & $0.023( \pm 0.002)$ & $0.09( \pm 0.02)$ \\
\hline G170.00-16.14-H2 & $2.36( \pm 0.03)$ & $0.06( \pm 0.01)$ & $0.022( \pm 0.002)$ & $14.19( \pm 1.35)$ & $0.019( \pm 0.002)$ & $0.06( \pm 0.01)$ \\
\hline G170.13-16.06-H1 & $1.61( \pm 0.04)$ & $0.13( \pm 0.01)$ & $0.085( \pm 0.012)$ & $3.11( \pm 0.44)$ & $0.084( \pm 0.012)$ & $0.13( \pm 0.01)$ \\
\hline G170.26-16.02-H2 & $2.30( \pm 0.01)$ & $0.12( \pm 0.01)$ & $0.027( \pm 0.001)$ & $10.41( \pm 0.33)$ & $0.024( \pm 0.001)$ & $0.12( \pm 0.01)$ \\
\hline G170.83-15.90-H1 & $0.72( \pm 0.01)$ & $0.13( \pm 0.03)$ & $0.049( \pm 0.004)$ & $1.95( \pm 0.26)$ & $0.048( \pm 0.004)$ & $0.13( \pm 0.03)$ \\
\hline G170.83-15.90-H2 & $0.96( \pm 0.01)$ & $0.18( \pm 0.09)$ & $0.032( \pm 0.002)$ & $3.47( \pm 0.38)$ & $0.030( \pm 0.002)$ & $0.18( \pm 0.09)$ \\
\hline G170.99-15.81-H1 & $1.36( \pm 0.01)$ & $0.26( \pm 0.05)$ & $0.050( \pm 0.002)$ & $3.16( \pm 0.22)$ & $0.049( \pm 0.002)$ & $0.26( \pm 0.05)$ \\
\hline G171.49-14.90-H1 & $3.79( \pm 0.03)$ & $0.11( \pm 0.01)$ & $0.025( \pm 0.001)$ & $18.11( \pm 0.56)$ & $0.022( \pm 0.001)$ & $0.11( \pm 0.01)$ \\
\hline G171.80-15.32-H1 & $2.61( \pm 0.04)$ & $0.10( \pm 0.01)$ & $0.031( \pm 0.002)$ & $10.65( \pm 0.73)$ & $0.029( \pm 0.002)$ & $0.10( \pm 0.01)$ \\
\hline G171.80-15.32-H2 & $1.37( \pm 0.01)$ & $0.17( \pm 0.08)$ & $0.027( \pm 0.002)$ & $5.96( \pm 0.72)$ & $0.024( \pm 0.002)$ & $0.16( \pm 0.08)$ \\
\hline G171.80-15.32-H3 & $1.91( \pm 0.01)$ & $0.23( \pm 0.03)$ & $0.030( \pm 0.001)$ & $7.13( \pm 0.28)$ & $0.028( \pm 0.001)$ & $0.23( \pm 0.03)$ \\
\hline $\mathrm{G} 171.91-15.65-\mathrm{H} 2^{\mathrm{e}}$ & $2.43( \pm 0.06)$ & $0.11( \pm 0.08)$ & $0.010( \pm 0.001)$ & $27.29( \pm 4.08)$ & $\ldots$ & $0.11( \pm 0.08)$ \\
\hline G171.91-15.65-H3 & $1.00( \pm 0.01)$ & $0.90( \pm 0.27)$ & $0.043( \pm 0.002)$ & $2.46( \pm 0.13)$ & $0.041( \pm 0.002)$ & $0.90( \pm 0.27)$ \\
\hline G172.06-15.21-H1 & $1.51( \pm 0.03)$ & $0.27( \pm 0.09)$ & $0.025( \pm 0.002)$ & $6.76( \pm 0.67)$ & $0.021( \pm 0.002)$ & $0.27( \pm 0.08)$ \\
\hline
\end{tabular}

Notes. All of these parameters come from a fit to a column density profile. The column density profiles are derived from a Herschel column density map. Due to the small size of G170.26-16.02-H1 and G171.91-16.15-H1, we cannot obtain their average column density distributions. Thus, we only present the parameters of 28 dense cores in this table.

${ }^{\mathrm{a}}$ The truncated radii are derived from fitting to a column density profile.

${ }^{\mathrm{b}}$ The central volume density as calculated by $n_{\mathrm{c}}=N_{\mathrm{c}} / 2 a \arctan (c), c \equiv R / a$.

c $a_{\mathrm{d}}$ represents the flat region that was removed by the beam effect using $a_{\mathrm{d}}=\left(a^{2}-\text { beam }^{2}\right)^{1 / 2}$.

${ }^{\mathrm{d}} R_{\mathrm{d}}$ represents the flat region that was removed by the beam effect using $R_{\mathrm{d}}=\left(R^{2}-\text { beam }^{2}\right)^{1 / 2}$.

${ }^{\mathrm{e}}$ The flat region $a$ of G171.91-15.65-core2 is smaller than the beam size $(0.012 \mathrm{pc})$, so we cannot calculate the $a_{\mathrm{d}}$.

where $N_{\mathrm{c}}, R_{\mathrm{t}}$, and $a$ are free parameters. Due to their small sizes, we cannot obtain average column density distributions for G170.26-16.02-H1 and G171.91-16.15-H1. Therefore, we only fit Herschel column density profiles to the other 28 cores in this section. $N_{\mathrm{c}}, R_{\mathrm{t}}, a$, and $n_{\mathrm{c}}$ are shown in Table 9, and the statistics of these parameters are summarized in Table 10.

The beam size is a critical factor that can affect the column density profile. For example, the resolution of the column density maps from Herschel data sets a lower limit for the size of the flat region $a$ and the core truncated radius $R_{\mathrm{t}}$. To remove the beam effect, we use a quadratic rule, $a_{\mathrm{d}}=\left(a^{2}-\theta_{\text {beam }}^{2}\right)^{1 / 2}$, to compute the deconvolved size of the flat region $a_{\mathrm{d}}$. A similar method was also used to calculate the deconvolved core radius $R_{\mathrm{d}}\left(a_{\mathrm{d}}\right.$ and $R_{\mathrm{d}}$ are listed in Table 9). Since the measured flat region of G171.91-15.65-H2 is smaller than the beam size, we cannot compute its $a_{\mathrm{d}}$, and that core was also excluded from further analyses. Therefore, we present the column density profiles of G171.91-15.65-H3 in Figure 9. The profiles for other dense cores are shown in the figure set.

The upper, middle, and bottom panels of Figure 10 present the average density profiles of starless cores, prestellar core candidates, and protostellar cores, respectively. We normalized each respective core type's column density profile before averaging. The truncated radius $R_{\mathrm{t}}$ decreases as dense cores evolve from starless to protostellar. The size of the central flat region $(a)$ is significantly larger in the starless cores (the mean value of $a$ is $0.48 \mathrm{pc}$ for starless cores), but is comparable between the prestellar candidates and protostellar cores (values of $a$ for both prestellar candidates and protostellar cores are $0.26 \mathrm{pc}$ ). This indicates that the starless cores are less peaked than prestellar core candidates or protostellar cores.

Figure 11 presents $R_{\mathrm{d}}, a_{\mathrm{d}}$, and $n_{\mathrm{c}}$. The upper panel of Figure 11 presents the results from fits to the column density profile for each core. The red, green, and blue dots are protostellar cores, prestellar core candidates, and starless cores, respectively. The bottom panel shows the values from fits to averaged protostellar cores, prestellar core candidates, and starless cores. According to the statistics of the fitting results (see Table 10), the denser the cores are, the smaller the values of $R_{\mathrm{d}}$ and $a$ are. In other words, the dense cores will show steeper density structures when they evolve from starless cores to prestellar or protostellar cores.

\subsection{CO Depletion}

Gaseous CO molecules can freeze out onto grain surfaces in cold and dense regions, and the $\mathrm{CO}$ molecules can return to the 
Table 10

Statistics of Parameters

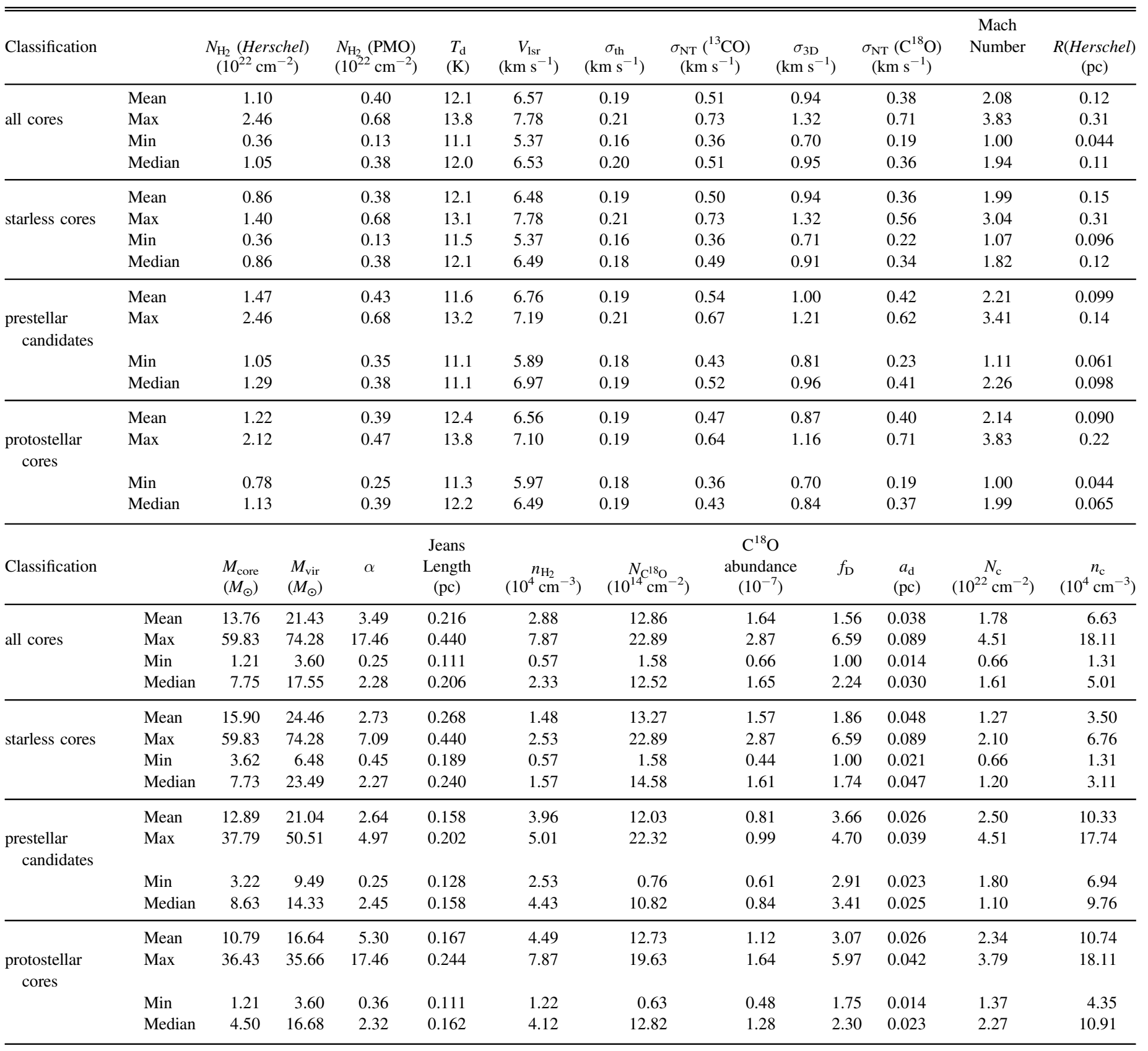

Note. The statistics of the main parameters for our analysis results. For all parameters, we represent the mean, maximum, minimum, and median values for all cores, starless cores, prestellar candidates, and protostellar cores, respectively. However, we have not included the SCUBA-2 data in this statistic, because only parts of the dense cores have been detected by SCUBA- 2 .

gas phase as the temperature rises due to heating from protostars (Bergin \& Langer 1997; Charnley 1997; Zhang et al. 2017).

Since $\mathrm{C}^{18} \mathrm{O}$ emission is generally optically thin, it is a more reliable tracer for $\mathrm{CO}$ depletion than ${ }^{12} \mathrm{CO}$ or ${ }^{13} \mathrm{CO}$. We calculate the core-averaged $\mathrm{C}^{18} \mathrm{O}$ abundance of each dense core as follows:

$$
X=\frac{N_{\mathrm{C}^{18} \mathrm{O}}}{N_{\mathrm{H}_{2}}(\text { Herschel })},
$$

where $N_{\mathrm{H}_{2}}$ is the core's Herschel-derived column density of $\mathrm{H}_{2}$ (listed in Table 3), and $N_{\mathrm{C}^{18} \mathrm{O}}$ is the $\mathrm{C}^{18} \mathrm{O}$ column density. It should be noted that the G166.99-15.34-H1 and G167.2315.32-H1 are located outside of the densest parts of the filament, and with exceptionally low $\mathrm{C}^{18} \mathrm{O}$ abundances. The low $\mathrm{C}^{18} \mathrm{O}$ abundance in these two cores may be attributed to the fact that they are less shielded from interstellar UV radiation and the $\mathrm{CO}$ molecules inside them are more easily dissociated. Thus, the $\mathrm{C}^{18} \mathrm{O}$ abundances of these two dense cores are very low, and they were excluded from fitting. In 
G171.91-15.65-H3

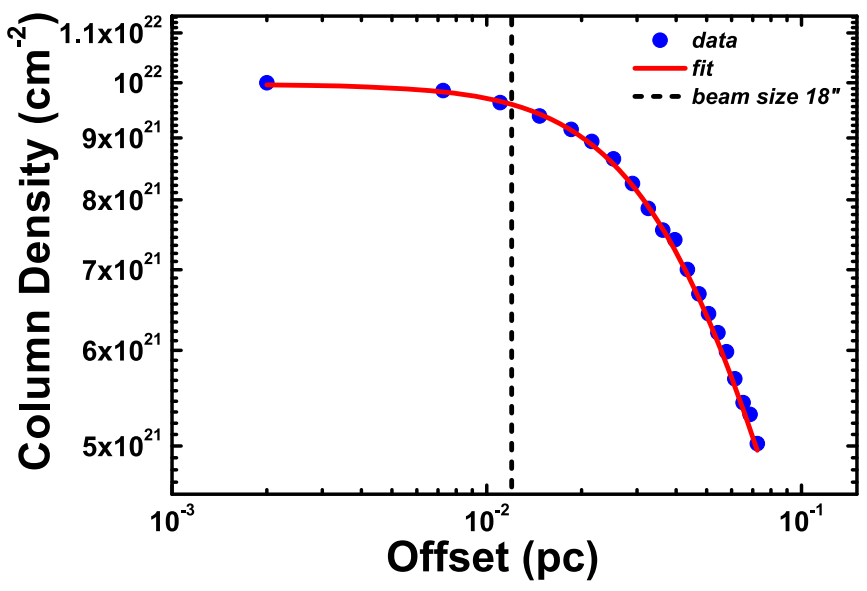

Figure 9. Column density profiles of the dense core for G171.91-15.65-H3 The red solid line represents the corresponding fit. The fitting results of $N_{\mathrm{c}}, R$, and $a$ are listed in Table 9. The flat central region and power-law decline can be seen in the figure. Due to its small size, we cannot obtain average column density distributions accurately for G170.26-16.02-H1 and G171.91-16.15-H1, and the flat region $a$ of G171.91-15.65-H1 is smaller than the beam size. Therefore, we only present the column density profile for G171.91-15.65-H3 in this section. The images for other sources are shown in the figure set.

(The complete figure set (27 images) is available.)

Figure 12, the red diamonds, green squares, and blue dots represent the protostellar cores, prestellar core candidates, and starless cores, respectively. $\mathrm{C}^{18} \mathrm{O}$ abundance is clearly anticorrelated with the column density, which also indicates that the $\mathrm{CO}$ depletion becomes more prevalent in denser cores.

In this work, we simply define a relative $\mathrm{CO}$ depletion factor $\left(f_{\mathrm{D}}\right)$ of dense cores as follows:

$$
f_{\mathrm{D}}=\frac{X_{\mathrm{max}}}{X_{\text {core }}}
$$

where $X_{\max }$ is the maximum $\mathrm{C}^{18} \mathrm{O}$ abundance in all the $\mathrm{L} 1495$ dense cores, and $X_{\text {core }}$ is the $\mathrm{C}^{18} \mathrm{O}$ abundance of a dense core. The $\mathrm{CO}$ depletion factors $\left(f_{\mathrm{D}}\right)$ range from 1 to $6.6( \pm 1.8)$, and for each core these parameters are listed in Table 7 . Please note that the $\mathrm{CO}$ depletion factors derived here should be treated as lower limits because we used core-averaged values. The $\mathrm{CO}$ depletion could be even more severe toward the core center.

Figure 13 presents $f_{\mathrm{D}}$ and the central volume densities $\left(n_{\mathrm{c}}\right)$ of the L1495 dense cores. There is a clear positive correlation between $f_{\mathrm{D}}$ and $n_{\mathrm{c}}$, indicating that $\mathrm{CO}$ depletion is more significant in denser cores. The starless cores are shown in the bottom left corner of Figure 13, thus these cores have the smallest $\mathrm{CO}$ depletion factors. $\mathrm{CO}$ depletion in prestellar candidates and protostellar cores is more significant than that in starless cores. The mean depletion factors of starless cores, prestellar core candidates, and protostellar cores are $1.9( \pm 0.7)$, $3.7( \pm 0.7)$, and $3.1( \pm 1.5)$, respectively. The prestellar candidates and protostellar cores are mixed with each other in Figures 12 and 13, indicating that the protostellar cores in L1495 are still at the earliest phases of star formation and thus have as a high degree of $\mathrm{CO}$ depletion as prestellar core candidates.

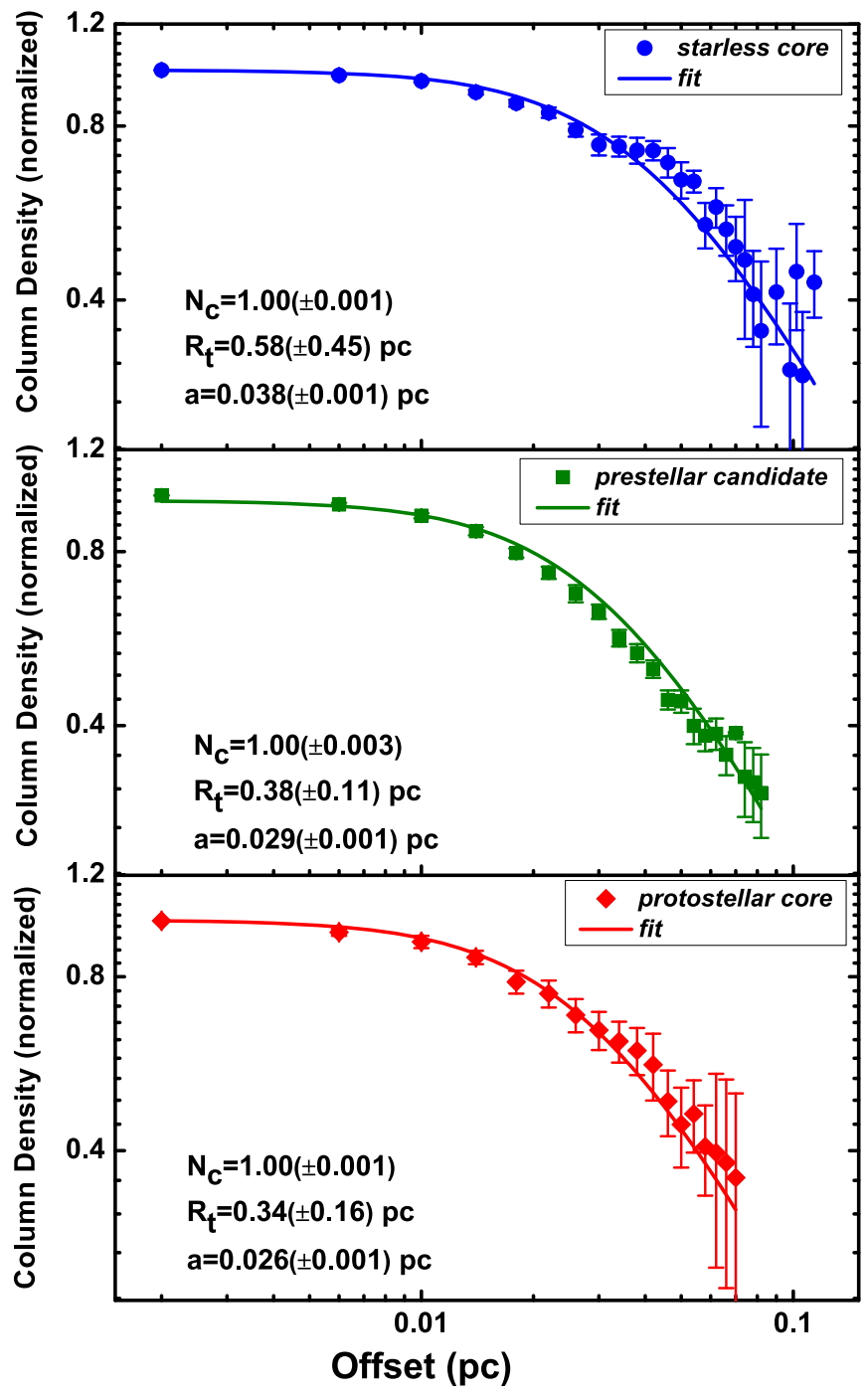

Figure 10. Average column density profile of starless cores (upper panel), prestellar core candidates (middle panel), and protostellar cores (bottom panel), respectively. The fitting results of $N_{\mathrm{c}}, R_{\mathrm{t}}$, and $a$ are shown in each panel.

\section{Summary}

To better understand the properties of PGCCs and dense cores in L1495 cloud, we have studied 16 dense clumps in the L1495 cloud with data from Herschel, JCMT/SCUBA-2, and the PMO $13.7 \mathrm{~m}$ telescope. The main findings of this work are as follows:

1. In the L1495 region of Taurus, we identified 30 dense cores in 16 PGCCs. The majority of Planck clumps have Herschel cores, often multiple, but only a subset of the Herschel cores have corresponding SCUBA-2 condensations. Based on SCUBA-2 and Herschel $70 \mu \mathrm{m}$ data, we have classified these 30 dense cores into three types: 15 are starless cores (i.e., with neither SCUBA-2 nor Herschel $70 \mu \mathrm{m}$ emission), 6 are prestellar core candidates (i.e., with SCUBA-2 emission but without Herschel $70 \mu \mathrm{m}$ emission) and 9 are protostellar cores (i.e., with Herschel $70 \mu \mathrm{m}$ emission). Our findings suggest that not all PGCCs contain prestellar objects. In general, however, the dense cores in PGCCs are usually at early evolutionary stages. 


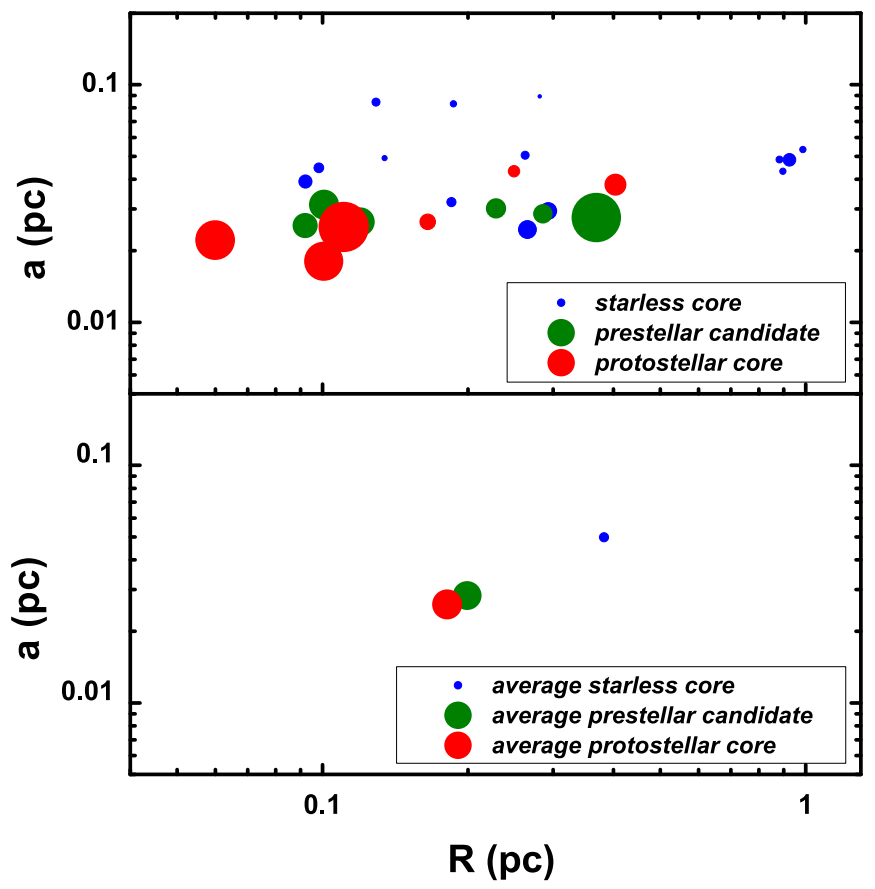

Figure 11. Top: the relationships between $R_{\mathrm{d}}, a_{\mathrm{d}}$, and $n_{\mathrm{c}}$ for L1495 dense cores The $x$ axis represents the radius $R_{\mathrm{d}}$, and the $y$ axis represents the flat region $a_{\mathrm{d}}$. The relative central volume densities $n_{\mathrm{c}}$ ranging from $1.3 \times 10^{4}$ to $1.8 \times 10^{5} \mathrm{~cm}^{-3}$ are shown by the sizes of the data points. Bottom: the relationships between $R_{\mathrm{d}}, a_{\mathrm{d}}$, and $n_{\mathrm{c}}$ of averaged protostellar cores, prestellar core candidates, and starless cores. In both panels, the red, green, and blue dots represent protostellar cores, prestellar core candidates, and starless cores, respectively.

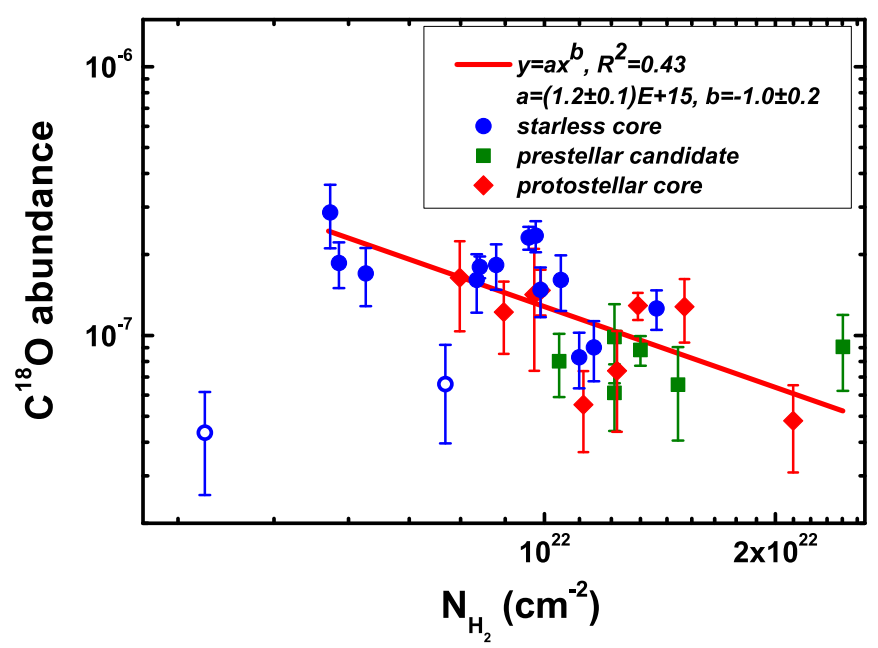

Figure 12. Correlation of $\mathrm{C}^{18} \mathrm{O}$ abundance and Herschel $N_{\mathrm{H}_{2}}$. The red line is a power-law fit. The red diamonds, green squares, and blue dots represent the protostellar cores, prestellar core candidates, and starless cores, respectively. The blue circles represent G166.99-15.34-H1 and G167.23-15.32-H1, and they are located outside of the densest parts of the filament. Thus, we excluded them from fitting. The function and coefficients of models are presented in the upperright corner of the image. A potential anti-correlation is found in the figure.

2. Based on Herschel data, the mean volume densities of the starless cores, prestellar core candidates and protostellar cores are $1.0( \pm 0.4) \times 10^{4} \mathrm{~cm}^{-3} \mathrm{~cm}^{-3}, 2.5( \pm 0.6) \times$ $10^{4} \mathrm{~cm}^{-3}$, and $2.7( \pm 1.0) \times 10^{4}$, respectively. The mean dust temperatures of the starless cores, prestellar core candidates and protostellar cores are $12.1( \pm 0.4) \mathrm{K}, 11.6$

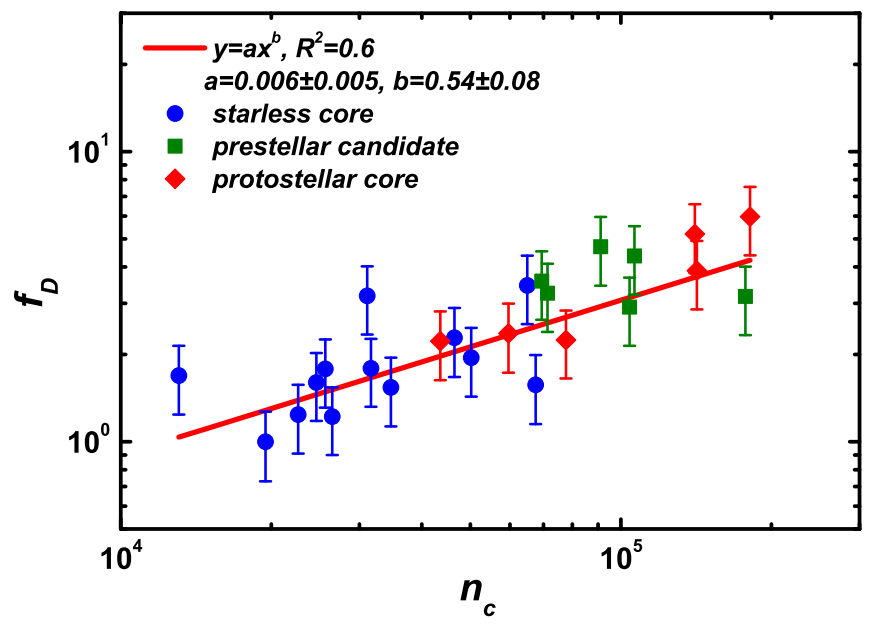

Figure 13. Correlation of relative depletion factors $\left(f_{\mathrm{D}}\right)$ and the central volume densities $n_{\mathrm{c}}$ (Herschel). The red line is a power-law fit, and blue dots, green squares, and red diamonds represent starless cores, prestellar core candidates, and protostellar cores, respectively.

$( \pm 0.8) \mathrm{K}$, and $12.4( \pm 0.7) \mathrm{K}$, respectively. On average, prestellar core candidates have slightly lower dust temperature and higher density than starless cores. The 9 protostellar cores appear to still be at the earliest protostellar evolutionary phases and the newly formed protostars have not significantly heated their envelopes.

3. Non-thermal and thermal velocity dispersions have been derived from the PMO data. In all 30 dense cores, the Mach numbers are all larger than 1 , with an average value of 2.1 $( \pm 0.8)$. Hence, the turbulence in the L1495 dense cores is supersonic, and all 30 dense cores may be turbulence-dominated.

4. The virial masses $\left(M_{\mathrm{vir}}\right)$ derived from the $\mathrm{C}^{18} \mathrm{O}$ data range from 3.6 $( \pm 0.7) M_{\odot}$ to $74.3( \pm 7.6) M_{\odot}$. The virial parameters $(\alpha)$ range from $0.3( \pm 0.1)$ to $17.5( \pm 7.3)$. Fourteen dense cores have virial parameters smaller than 2 , and 18 dense cores have virial parameters larger than 2 . The virial parameter and core mass follow the power-law trend, $\alpha=$ $\alpha_{\min } \cdot\left(M_{\text {core }} / M_{\max }\right)^{h_{\alpha}}$, with $h_{\alpha}=-0.74( \pm 0.08)$.

5 . The column density profiles of 28 dense cores have been derived from Herschel data. The central volume density $\left(n_{\mathrm{c}}\right)$, the size of the flat region $(a)$, and the truncated radius $\left(R_{\mathrm{t}}\right)$ were obtained by fitting a column density profile. We found that the values of flat region size and truncated radius decrease as $n_{\mathrm{c}}$ increases. This indicates that dense cores shrink as they evolve from starless cores to protostellar cores (Ward-Thompson et al. 1994).

6. The $\mathrm{C}^{18} \mathrm{O}$ abundances are used to investigate the $\mathrm{CO}$ depletion degree in three types of dense cores. The mean $\mathrm{C}^{18} \mathrm{O}$ abundances of protostellar cores, prestellar core candidates, and starless cores are $1.12( \pm 0.48) \times 10^{-7}$, $8.07( \pm 1.34) \times 10^{-8}$ and $1.57( \pm 0.65) \times 10^{-7}$, respectively. This variation means that $\mathrm{CO}$ depletion in prestellar core candidates is most significant. The coreaveraged $\mathrm{CO}$ depletion factors $\left(f_{\mathrm{D}}\right)$ range from 1 to 6.6 $( \pm 1.8)$. Our results support the idea that the $\mathrm{C}^{18} \mathrm{O}$ abundance can be used as an evolutionary tracer for molecular cloud cores, as suggested by Caselli et al. (1999), Di Francesco et al. (2007), and Liu et al. (2013). 
S.-L. Qin is supported by the National Key R\&D Program of China (NO. 2017YFA0402701), by the Joint Research Fund in Astronomy (U1631237) under cooperative agreement between the National Natural Science Foundation of China (NSFC) and the Chinese Academy of Sciences (CAS), and by the Top Talents Program of Yunnan Province (2015HA030). Tie Liu is supported by a KASI fellowship and a EACOA fellowship. Ke Wang is supported by grant WA3628-1/1 of the German Research Foundation (DFG) through the priority program 1573 ("Physics of the Interstellar Medium"). This research was partly supported by the OTKA grant NN-111016. We thank Michel Fich, Mika Juvela, Jan Wouterloot, Archana Soam, M. R. Cunningham, Chang Won Lee, Paul F. Goldsmith, A. RiveraIngraham, Jinghua Yuan, Pak Shing Li, Johanna Malinen, George J. Bendo, Hong-Li Liu, Miju Kang, Neal J. Evans II, Patricio Sanhueza, Edith Falgarone, Glenn J. White, Izaskun Jimenez-Serra, You-Hua Chu, Yao-Lun Yang, JinHua He, and Hauyu Baobab Liu for helpful discussions and comments. The James Clerk Maxwell Telescope is operated by the East Asian Observatory on behalf of The National Astronomical Observatory of Japan, Academia Sinica Institute of Astronomy and Astrophysics, the Korea Astronomy and Space Science Institute, the National Astronomical Observatories of China and the Chinese Academy of Sciences (grant No. XDB09000000), with additional funding support from the Science and Technology Facilities Council of the United Kingdom and participating universities in the United Kingdom and Canada. Additional funds for the construction of SCUBA-2 were provided by the Canada Foundation for Innovation. This research has made use of data from the Herschel Gould Belt survey (HGBS) project (http://gouldbelt-herschel.cea.fr). The HGBS is a Herschel Key Programme jointly carried out by SPIRE Specialist Astronomy Group 3 (SAG 3), scientists of several institutes in the PACS Consortium (CEA Saclay, INAF-IFSI Rome and INAF-Arcetri, KU Leuven, MPIA Heidelberg), and scientists of the Herschel Science Center (HSC).

\section{ORCID iDs}

Mengyao Tang (i) https://orcid.org/0000-0001-9160-2944

Tie Liu (1) https://orcid.org/0000-0002-5286-2564

Sheng-Li Qin (1) https://orcid.org/0000-0003-2302-0613

Kee-Tae Kim (1) https://orcid.org/0000-0003-2412-7092

Yuefang Wu (1) https://orcid.org/0000-0002-5076-7520

Ken'ichi Tatematsu (1) https://orcid.org/0000-0002-8149-8546

Ke Wang (i) https://orcid.org/0000-0002-7237-3856

Harriet Parsons (i) https://orcid.org/0000-0002-6327-3423

Patrick M. Koch (1) https://orcid.org/0000-0003-2777-5861

Patricio Sanhueza (다 https://orcid.org/0000-0002-7125-7685

D. Ward-Thompson (i) https://orcid.org/0000-0003-

1140-2761

James Di Francesco (다 https://orcid.org/0000-0002-9289-2450

Chuan-Peng Zhang $\odot$ https://orcid.org/0000-0002-4428-3183

\section{References}

André, P., Di Francesco, J., Ward-Thompson, D., et al. 2014, in Protostars and Planets VI, ed. H. Beuther et al. (Tucson, AZ: Univ. Arizona Press), 27 André, Ph., Men'shchikov, A., Bontemps, S., et al. 2010, A\&A, 518, L102 Bergin, E. A., \& Langer, W. D. 1997, ApJ, 486, 316

Bertoldi, F., \& Mckee, C. F. 1992, ApJ, 395, 140
Bintley, D., Holland, W. S., MacIntosh, M. J., et al. 2014, Proc. SPIE, 9153, 915303

Buckle, J. V., Drabek-Maunder, E., Greaves, J., et al. 2015, MNRAS, 449, 2472

Caselli, P. 2011, in IAU Symp. 280, The Molecular Universe, ed. J. Cernicharo \& R. Bachiller (Cambridge: Cambridge Univ. Press), 19

Caselli, P., Walmsley, C. M., Tafalla, M., Dore, L., \& Myers, P. C. 1999, ApJL, 523, L165

Chackerian, C., \& Tipping, R. H. 1983, JMoSp, 99, 431

Chapin, E., Gibb, A. G., Jenness, T., et al. 2013, StaUN, 258

Charnley, S. B. 1997, MNRAS, 291, 455

Currie, M. J., Berry, D. S., Jenness, T., et al. 2014, in ASP Conf. Ser. 485, adass XXIII, ed. N. Manset \& P. Forshay (San Francisco, CA: ASP), 391

Dapp, W. B., \& Basu, Shantanu. 2009, MNRAS, 395, 1092D

Dempsey, J. T., Friberg, P., Jenness, T., et al. 2013, MNRAS, 430, 2534D

Di Francesco, J., Evans, N. J., II, Caselli, P., et al. 2007, in Protostars and Planets V, ed. B. Reipurth, D. Jewitt, \& K. Keil (Tucson, AZ: Univ. Arizona Press), 17

Elias, J. H. 1987, ApJ, 224, 857

Fehér, O., Juvela, M., Lunttila, T., et al. 2017, A\&A, 606, A102

Friesen, R. K., Bourke, T. L., Di Francesco, J., Gutermuth, R., \& Myers, P. C. 2016, ApJ, 833, 204

Garden, R. P., Hayashi, M., Hasegawa, T., Gatley, I., \& Kaifu, N. 1991, ApJ, 374,540

Giannetti, A., Wyrowski, F., Csengeri, T., et al. 2014, A\&A, 570, A65

Hacar, A., Tafalla, M., Kauffmann, J., \& Kovács, A. 2013, A\&A, 554, A55

Hawkins, I., \& Jura, M. 1987, ApJ, 317, 926

Hildebrand, R. H. 1983, QJRAS, 24, 267

Holland, W. S., Bintley, D., Chapin, E. L., et al. 2013, MNRAS, 430, 2513

Jeans, J. H. 1928, Astronomy and Cosmogony (Cambridge: Cambridge Univ. Press)

Juvela, M., He, J., Pattle, K., et al. 2017, A\&A, in press (arXiv:1711.09425)

Kauffmann, J., Bertoldi, F., Bourke, T. L., Evens, N. J., II, \& Lee, C. W. 2008 A\&A, 487, 993

Kauffmann, J., Pillai, T., \& Goldsmith, P. F. 2013, ApJ, 779, 185

Kenyon, S. J., Dobrzycka, D., \& Hartmann, L. 1994, AJ, 108, 1872

Kim, J., Lee, J.-E., Liu, T., et al. 2017, ApJS, 231, 9

Kirk, J. M., Ward-Thompson, D., Palmeirim, P., et al. 2013, MNRAS, 432, $1424 \mathrm{~K}$

Könyves, V., André, Ph., Men'shchikov, A., et al. 2015, A\&A, 584, A91

Liu, T., Kim, K.-T., Juvela, M., et al. 2018, ApJS, 234, 28

Liu, T., Wu, Y., \& Mardones, D. 2014, PKAS, 30, 79L

Liu, T., Wu, Y., \& Zhang, H. 2012, ApJS, 202, 4

Liu, T., Wu, Y., \& Zhang, H. 2013, ApJL, 775, L2

Liu, T., Zhang, Q., Kim, K.-T., et al. 2016, ApJS, 222, 7

Loinard, L., Torres, R. M., Mioduszewski, A. J., \& Rodríguez, L. F. 2008, RMxAC, 34, 14

Loren, R. B. 1989, ApJ, 338, 925

Mac Low, M.-M., \& Klessen, R. S. 2004, RvMP, 76, 125

MacLaren, I., Richardon, K. M., \& Wolfendale, A. W. 1988, ApJ, 333, 821

Mairs, S., Johnstone, D., Kirk, H., et al. 2015, MNRAS, 454, 2557

Marsh, K. A., Griffin, M. J., Palmeirim, P., et al. 2014, MNRAS, 439, 3683M

Marsh, K. A., Kirk, J. M., André, Ph., et al. 2016, MNRAS, 459, 342

Meng, F., Wu, Y., \& Liu, T. 2013, ApJS, 209, 37

Ohashi, S., Sanhueza, P., Chen, H.-R. V., et al. 2016, ApJ, 833, 209

Onishi, T., Mizuno, A., Kawamura, A, Ogawa, H., \& Fukui, Y. 1996, ApJ, 465,815

Onishi, T., Mizuno, A., Kawamura, A., Tachihara, K., \& Fukui, Y. 2002, ApJ, 575,950

Pattle, K., Ward-Thompson, D., Kirk, J. M., et al. 2015, MNRAS, 450, 1094 Penzias, A. A. 1980, Sci, 208, 663

Pilbratt, G. L., Riedinger, J. R., Passvogel, T., et al. 2010, A\&A, 518, L1

Planck Collaboration, Ade, P. A. R., Aghanim, N., et al. 2011, A\&A, 536, 23

Planck Collaboration, Ade, P. A. R., Aghanim, N., et al. 2016, A\&A, 594, A28

Sanhueza, P., Jackson, J. M., Zhang, Q., et al. 2017, ApJ, 841, 97

Sault, R. J., Teuben, P. J., \& Wright, M. C. 1995, in ASP Conf. Ser. 77, adass IV, ed. R. A. Shaw, H. E. Payne, \& J. J. E. Hayes (San Francisco, CA: ASP), 433

Schmalzl, M., Kainulainen, J., Quanz, S. P., et al. 2010, ApJ, 725, 1327

Seo, Y. M., Shirley, Y. L., Goldsmith, P., et al. 2015, ApJ, 805, 185S

Shan, W., Yang, Y., Shi, S., et al. 2012, ITTST, 2, 593

Straizys, V., \& Meistas, E. 1980, AcA, 30, 541

Tachihara, K., Mizuno, A., \& Fukui, Y. 2000, ApJ, 528, 817

Tatematsu, K., Liu, T., Ohashi, S., et al. 2017, ApJS, 228, 12

Torres, R. M., Loinard, L., Mioduszewski, A. J., et al. 2012, ApJ, 747, 18T 
Tóth, L. V., Marton, G., Zahorecz, S., et al. 2014, PASJ, 66, 17

Tóth, L. V., Zahorecz, S., Marton, G., et al. 2017, in IAU Symp. 316, Formation, Evolution, and Survival of Massive Star Clusters, ed. C. Charbonnel \& A. Nota (Cambridge: Cambridge Univ. Press), 133

Tóth, L. V., Zahorecz, S., Marton, G., et al. 2016, in IAU Symp. From Interstellar Clouds to Star-forming Galaxies, ed. P. Jablonka, P. André, \& F. van der Tak (Cambirdge: Cambridge Univ. Press), E75

Van der Tak, F. F. S., Black, J. H., Schöier, F. L., Jansen, D. J., \& van Dishoeck, E. F. 2007, A\&A, 468, 627

Ward-Thompson, D., Di Francesco, J., Hatchell, J., et al. 2007, PASP, 119, 855
Ward-Thompson, D., Pattle, K., Kirk, J. M., et al. 2016, MNRAS, 463, 1008 Ward-Thompson, D., Scott, P. E., Hills, R. E., \& André, P. 1994, MNRAS, 268, 276

Wu, Y., Liu, T., Meng, F., et al. 2012, ApJ, 756, 76

Yuan, J., Wu, Y., Liu, T., et al. 2016, ApJ, 820, 37

Zahorecz, S., Jimenez-Serra, I., Wang, K., et al. 2016, A\&A, 591, A105

Zhang, C.-P., Yuan, J.-H., Li, G.-X., Zhou, J.-J., \& Wang, J.-J. 2017, A\&A, 598, A76

Zhang, Q., Wang, K., Lu, X., \& Jiménez-Serra, I. 2015, ApJ, 804, 141

Zhang, T., Wu, Y., Liu, T., et al. 2016, ApJS, 224, 43 